\title{
Cinnamylindoline Derivatives: Synthesis and Factor Xa (FXa) Inhibitory Activities ${ }^{1)}$
}

\author{
Tetsuji Noguchi, ${ }^{a}$ Naoki TanakA ${ }^{*}, b$ Toyoki Nishimata, ${ }^{b}$ Riki Goto, ${ }^{b}$ Miho Hayakawa, ${ }^{a}$ \\ Atsuhiro Sugidachi, ${ }^{c}$ Taketoshi OGawA, ${ }^{d}$ Fumitoshi Asai, ${ }^{c}$ and Koichi Fujmoto ${ }^{b, 2)}$ \\ ${ }^{a}$ Medicinal Chemistry Research Laboratories II, Daiichi Sankyo Co., Ltd.; 1-16-13 Kitakasai, Edogawa-ku, Tokyo \\ 134-8630, Japan: ${ }^{b}$ Medicinal Chemistry Research Laboratories I, Daiichi Sankyo Co., Ltd.; ${ }^{c}$ Biological Research \\ Laboratories II, Daiichi Sankyo Co., Ltd.; and ${ }^{d}$ Biological Research Laboratories I, Daiichi Sankyo Co., Ltd.; 1-2-58 \\ Hiromachi, Shinagawa-ku, Tokyo 140-8710, Japan.
}

Received June 21, 2007; accepted August 10, 2007; published online August 10, 2007

A series of cinnamylindoline derivatives were synthesized, and their factor Xa (FXa) inhibitory activities and selectivity over trypsin were evaluated. Among them, some novel derivatives showed potent FXa inhibitory activities and good selectivity over trypsin. Especially, (E)-2-\{5-[1-(acetimidoyl)piperidin-4-yloxy]-2-[2-(5amidino-2-hydroxyphenyl)ethen-1-yl]indolin-1-ylsulfonyl \}acetic acid (22f) having 2-hydroxycinnamyl moiety exhibited the most potent FXa inhibitory activity in vitro. Furthermore, $22 \mathrm{f}$ also exhibited potent anticoagulant activities in vitro.

Key words factor Xa inhibitory activity; cinnamylindoline derivative; anticoagulant

In recent days, factor $\mathrm{Xa}(\mathrm{FXa})$ inhibitor attracts much attention as a promising drug candidate for anticoagulation. ${ }^{3)}$ $\mathrm{FXa}$, a serine protease in the blood coagulation cascade, ${ }^{4)}$ is essential for the formation of thrombin. It plays an important role in the coagulation cascade at the convergent point of the intrinsic and the extrinsic pathway. FXa inhibitor is expected to be a novel antithrombotic with potential for the treatment and prevention of thromboembolic diseases. ${ }^{5,6)}$

In previous papers, ${ }^{7,8)}$ we reported the syntheses and FXa inhibitory activities of bisamidine compounds having indoline moiety in the center of the molecule. In these studies, we found that $(R)-5$-[1-(acetimidoyl)piperidin-4-yloxy]-2-(7amidinonaphthalen-2-yl)-1-(ethanesulfonyl)indoline $((R)-1)$ and $(\{(R)-5$-[1-(acetimidoyl)piperidin-4-yloxy]-2-(7-amidinonaphthalen-2-yl)indolin-1-yl $\}$ sulfonyl)acetic acid $((R)-2)$ exhibited potent FXa inhibitory activities (Fig. 1). However, these compounds also exhibited potent inhibitory activities

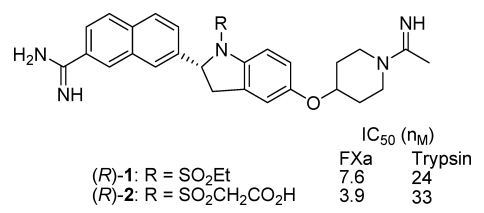

Fig. 1. Structures of Naphthylindoline Derivatives against trypsin which belongs to a serine protease family. In general, compounds having selective inhibitory activity against target enzyme were favorable as drug candidates because of less possibility of unexpected adverse reaction. From this viewpoint, we searched for a potent and selective FXa inhibitor.

Herein, we describe the synthesis and structure-activity relationships (SARs) and FXa selectivity of these compounds.

\section{Chemistry}

(E)-Substituted cinnamaldehyde intermediates $\mathbf{4 a}-\mathbf{e}$ were synthesized as shown in Chart 1. 3-Cyanobenzaldehyde (3) was reacted with ylides to give corresponding substituted cinnamaldehyde $\mathbf{4 a -}$ - c. Compound $\mathbf{3}$ and methyl acrylate (5) were coupled to give cinnamate $\mathbf{6 d}$ having a methoxymethyl group on the $\alpha$-position. 3-Cyanoacetophenone (7) was reacted with an ylide to give cinnamate 6e having a methyl group on the $\beta$-position. Compounds $\mathbf{6 d}$ and $\mathbf{6 e}$ were converted to cinnamaldehydes $\mathbf{4 d}$ and $\mathbf{4 e}$ by 3 steps, respectively.

The syntheses of bisamidine derivatives $(\mathbf{1 3 a}-\mathbf{e})$ are outlined in Chart 2. Cinnamaldehyde $\mathbf{4 a -}$ - were coupled with compound $\mathbf{8} \mathbf{a}^{7)}$ by tetrabutylammonium fluoride (TBAF) to give the corresponding alcohols $9 \mathbf{a}-\mathbf{e}$. ${ }^{9)}$ Nitro groups of alcohols $9 \mathbf{a}-\mathbf{e}$ were converted to ethanesulfonylamino groups

4a: $\mathrm{R}^{1}=\mathrm{H}, \mathrm{R}^{2}=\mathrm{H}, 57 \%$ 4b: $R^{1}=M e, R^{2}=H, 76 \%$ 4c: $R^{1}=E t, R^{2}=H, 38 \%$
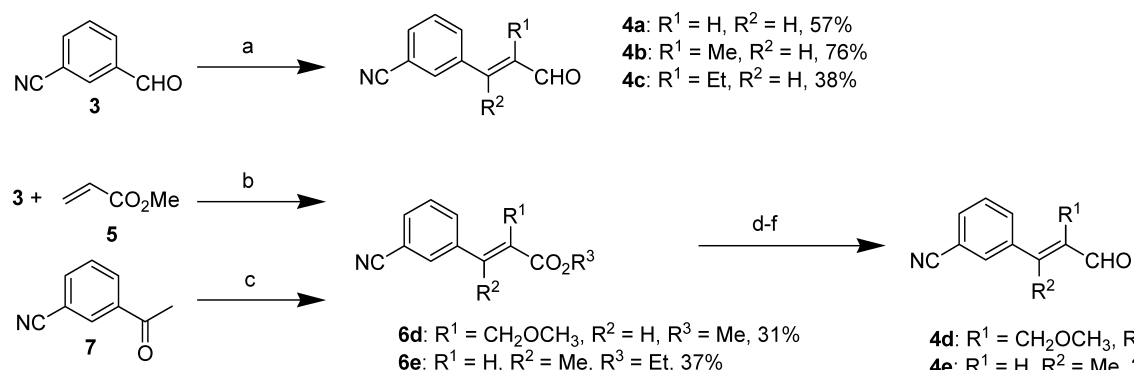

6d: $\mathrm{R}^{1}=\mathrm{CH}_{2} \mathrm{OCH}_{3}, \mathrm{R}^{2}=\mathrm{H}, \mathrm{R}^{3}=\mathrm{Me}, 31 \%$ 6e: $R^{1}=H, R^{2}=M e, R^{3}=E t, 37 \%$ 4d: $\mathrm{R}^{1}=\mathrm{CH}_{2} \mathrm{OCH}_{3}, \mathrm{R}^{2}=\mathrm{H}, 41 \%$ 4e: $R^{1}=H, R^{2}=M e, 24 \%$ 


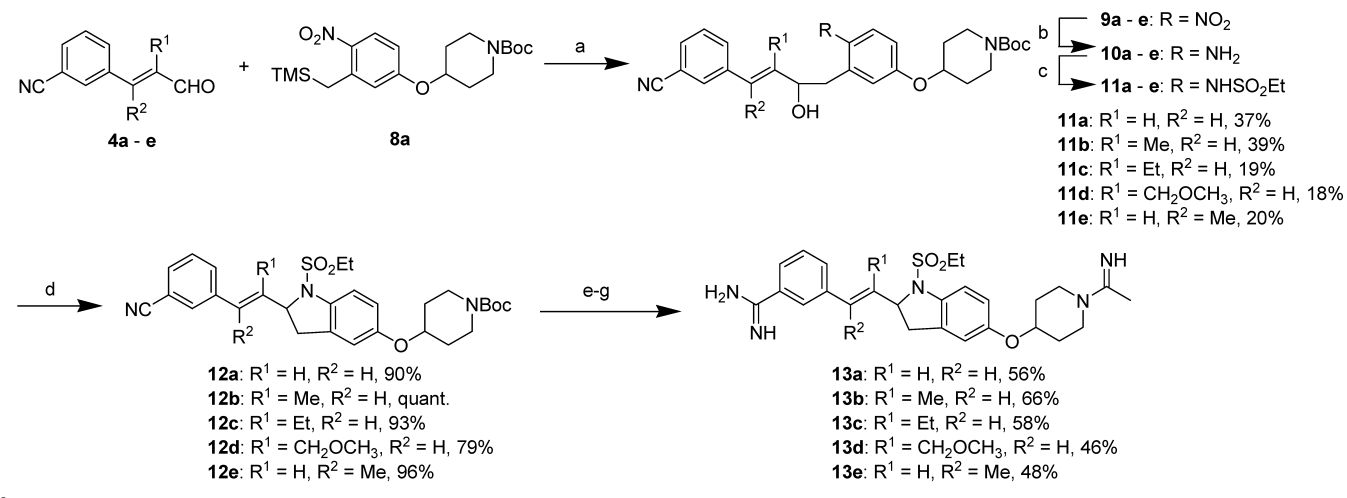

${ }^{a}$ Reagents : a) TBAF / THF; b) $\mathrm{Zn} / \mathrm{AcOH}$; c) EtSO ${ }_{2} \mathrm{Cl}$, Pyr. / $\mathrm{CH}_{2} \mathrm{Cl}_{2}$; d) $n$ - $\mathrm{Bu}_{3} \mathrm{P}, \mathrm{ADDP} / \mathrm{THF}$; e) $\mathrm{HCl}$ g. / EtOH-CH $\mathrm{Cl}_{2}$; f) $\mathrm{NH}_{3}$ aq., NH $\mathrm{NH}_{4} \mathrm{Cl} / \mathrm{EtOH}-\mathrm{H}_{2} \mathrm{O}$; g) $\mathrm{Et}_{3} \mathrm{~N}$, ethyl acetimidate hydrochloride / $\mathrm{EtOH}$

Chart 2

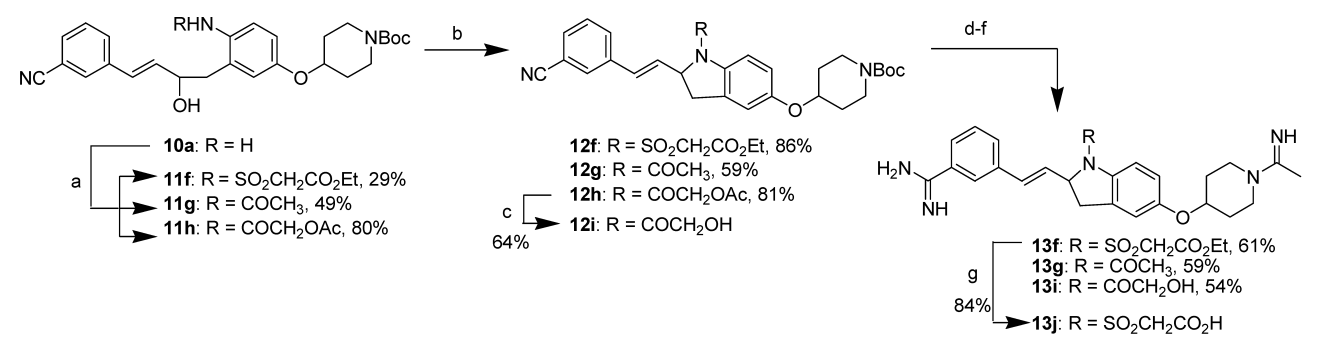

a Reagents : a) RCl, Pyr. / $\mathrm{CH}_{2} \mathrm{Cl}_{2}$; b) $n-\mathrm{Bu}_{3} \mathrm{P}, \mathrm{ADDP} / \mathrm{THF}$; c) $\mathrm{K}_{2} \mathrm{CO}_{3} / \mathrm{MeOH}$; d) $\mathrm{HCl}$ g. / EtOH- $\mathrm{CH}_{2} \mathrm{Cl}_{2}$; e) $\mathrm{NH}_{3}$ aq., $\mathrm{NH}_{4} \mathrm{Cl} /$ EtOH- $\mathrm{H}_{2} \mathrm{O}$; f) Et $\mathrm{Et}_{3} \mathrm{~N}$, ethyl acetimidate hydrochloride / EtOH; g) $4 \mathrm{~N} \mathrm{HCl}$

Chart 3

Chart 3
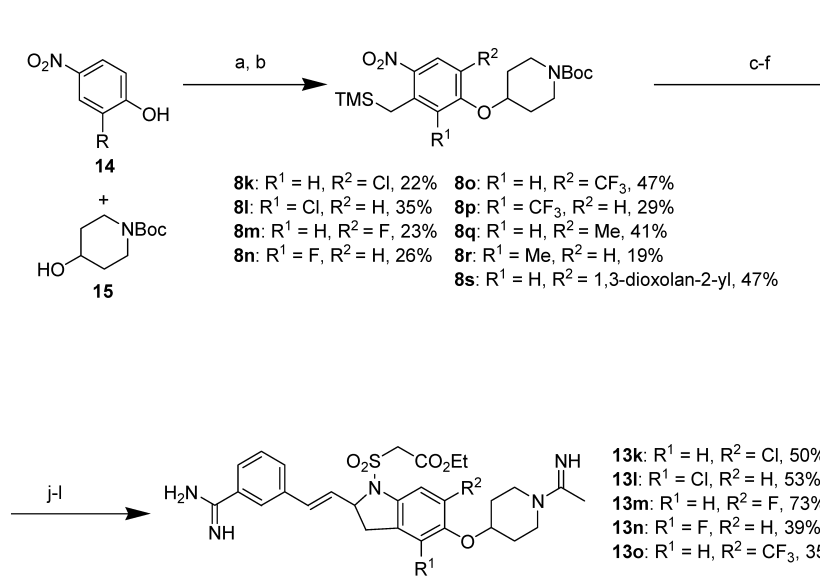

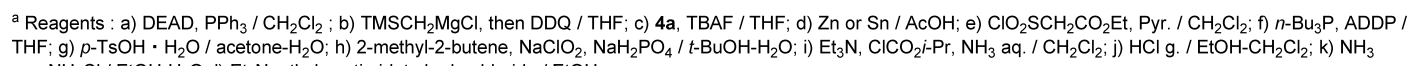
aq., $\mathrm{NH}_{4} \mathrm{Cl} / \mathrm{EtOH}-\mathrm{H}_{2} \mathrm{O}$; l) $\mathrm{Et} 3 \mathrm{~N}$, ethyl acetimidate hydrochloride / $\mathrm{EtOH}$

\begin{abstract}
3k: $\mathrm{R}^{1}=\mathrm{H}, \mathrm{R}^{2}=\mathrm{Cl}, 50 \%$ 3I: $R^{1}=C_{1} R^{2}=H, 50 . R^{1}=C_{3}, R^{2}=\mathrm{H}, 48 \%$ 3m: $R^{1}=H, R^{2}=H, 53 \% \quad$ 13q: $R^{1}=H, R^{2}=M e, 55 \%$ 13r: $R^{1}=M e, R^{2}=H, 34 \%$ 13t: $R^{1}=\mathrm{H}, \mathrm{R}^{2}=\mathrm{CO}_{2} \mathrm{Et}, 27 \%$ 13o: $R^{1}=H, R^{2}=C_{3}, 35 \%$ 13u: $R^{1}=H, R^{2}=C_{2} \mathrm{NH}_{2}, 32 \%$
\end{abstract} 12. $\mathrm{R}^{1}=\mathrm{H}, \mathrm{R}^{2}=\mathrm{Cl}, 8 \%$ 120: $\mathrm{R}^{1}=\mathrm{H}, \mathrm{R}^{2}=\mathrm{CF}_{3}, 21 \%$ 2m: $R^{1}=H, R^{2}=F, 13 \%$ 12q: $R^{1}=H, R^{2}=M e, 9 \%$

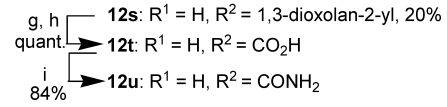

Chart 4

to give 11a-e. Intramolecular Mitsunobu reaction ${ }^{10)}$ of $11 \mathrm{a}-\mathrm{e}$ with $n-\mathrm{Bu}_{3} \mathrm{P}$ and $1,1^{\prime}$-(azodicarbonyl)dipiperidine (ADDP) afforded 12a-e having indoline rings. 12a-e were converted to desired bisamidine derivatives $13 \mathbf{a}-\mathbf{e}$ by 3 steps.

The syntheses of bisamidine derivatives (13f, 13g, 13i, 13j) are outlined in Chart 3. After reaction of aniline 10a with a sulfonyl chloride ${ }^{11)}$ or acyl chlorides, resulting sulfonamide $11 \mathrm{f}$ or amides $\mathbf{1 1 g}$ and $\mathbf{1 1 h}$ were cyclized by the same process described above to give indoline $\mathbf{1 2 f}-\mathbf{h}$. An acetoxy group of indoline $\mathbf{1 2 h}$ was hydrolyzed to give alcohol $\mathbf{1 2 i}$. In a similar method described above, 12f, $12 \mathrm{~g}$ and $\mathbf{1 2} \mathbf{i}$ were converted to desired bisamidine derivatives 13f, $\mathbf{1 3 g}$ and 13i, respectively. Carboxylic acid $\mathbf{1 3 \mathbf { j }}$ was synthesized from ester $\mathbf{1 3 f}$ by acid hydrolysis.

The syntheses of bisamidine derivatives $(\mathbf{1 3 k}-\mathbf{r}, \mathbf{1 3 t}$, 13u) having substituents on aryl carbons of indoline are outlined in Chart 4. Substituted nitrophenols 14 were coupled with $N$-protected piperidinol $\mathbf{1 5}$, followed by reaction with (trimethylsilylmethyl)magnesium chloride to give the corresponding 2-substituted-3-trimethylsilylmethyl derivatives (81, 8n, 8p, 8r) and/or 2-substituted-5-trimethylsilylmethyl derivatives $(\mathbf{8 k}, \mathbf{8 m}, \mathbf{8 0}, \mathbf{8 q}, \mathbf{8 s}){ }^{12)}$ Compounds $\mathbf{8 k}-\mathbf{s}$ were converted to cyclized compounds $12 \mathbf{k}-\mathbf{s}$ by a similar method described in Chart 2. A 1,3-dioxolan-2-yl group of compound 12s was converted to a carboxyl group (12t) by 2 


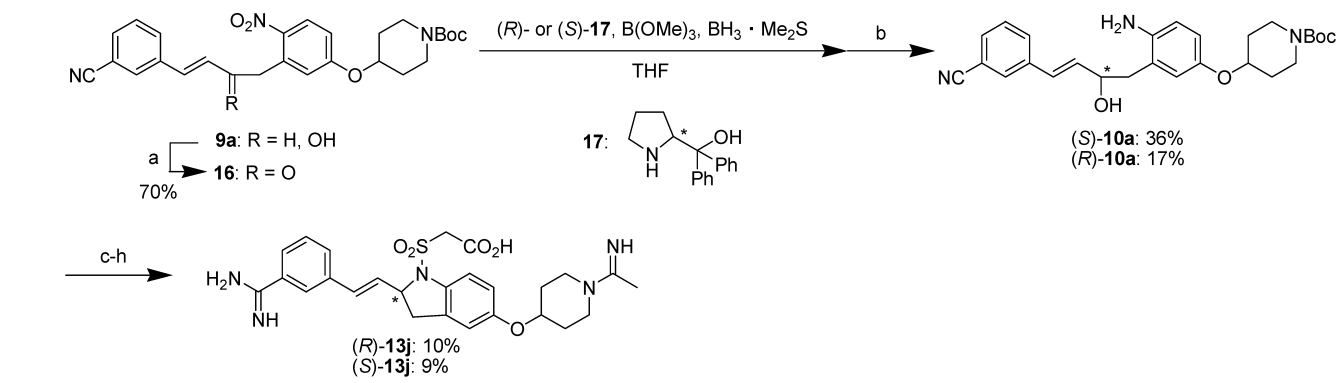

${ }^{a}$ Reagents : a) PCC / $\mathrm{CH}_{2} \mathrm{Cl}_{2}$; b) $\mathrm{Zn} / \mathrm{AcOH}$; c) $\mathrm{ClO}_{2} \mathrm{SCH}_{2} \mathrm{CO}_{2} \mathrm{Et}$, Pyr. / $\mathrm{CH}_{2} \mathrm{Cl}_{2}$; d) $\left.n-\mathrm{Bu}_{3} \mathrm{P}, \mathrm{ADDP} / \mathrm{THF} ; \mathrm{e}\right) \mathrm{HCl}$ g. / EtOH-CH $\mathrm{Et}_{3} \mathrm{~N}$, ethyl acetimidate hydrochloride / $\left.\mathrm{EtOH} ; \mathrm{h}\right){ }_{\mathrm{N}} \mathrm{HCl}$

Chart 5
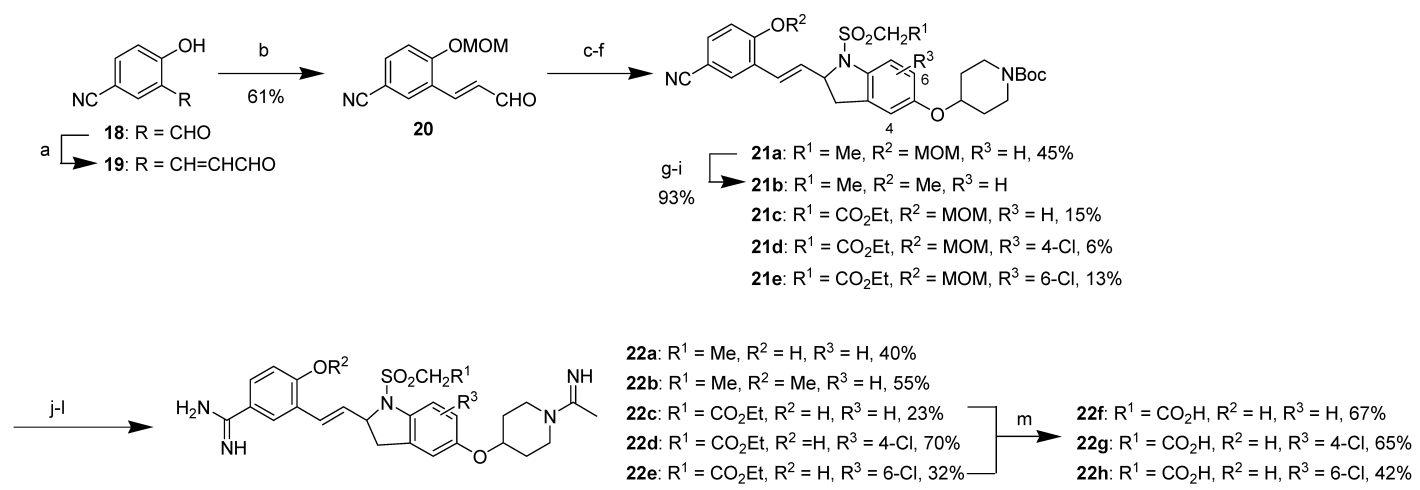

a Reagents : a) $\mathrm{Ph}_{3} \mathrm{P}=\mathrm{CHCHO} / \mathrm{PhCH}_{3}$; b) $\mathrm{MOMCl}, \mathrm{Et}_{3} \mathrm{~N} / \mathrm{DMA}$; c) $8 \mathrm{a}$ or $8 \mathrm{k}$ or $81, \mathrm{TBAF} / \mathrm{THF}$; d) $\mathrm{Zn} / \mathrm{AcOH} \cdot$ e) $\mathrm{R}^{1} \mathrm{CH}_{2} \mathrm{SO}_{2} \mathrm{Cl}$

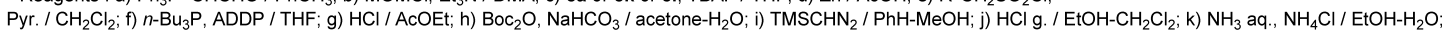
I) $\mathrm{Et}_{3} \mathrm{~N}$, ethyl acetimidate hydrochloride / $\left.\mathrm{EtOH} ; \mathrm{m}\right) 1 \mathrm{~N} \mathrm{HCl}$.

Chart 6

steps. Furthermore, $\mathbf{1 2 t}$ was converted to amide $\mathbf{1 2 u}$ in a standard manner. Cyclized compounds $12 \mathbf{k}-\mathbf{r}, \mathbf{1 2 t}$ and $12 \mathbf{u}$ were converted to desired bisamidine compounds $13 \mathbf{k}-\mathbf{r}$, 13t and 13u by a similar method described in Chart 2 .

The syntheses of optically active $(R)-\mathbf{1 3} \mathbf{j}$ and $(S) \mathbf{- 1 3} \mathbf{j}$ are outlined in Chart 5. After oxidation of racemic alcohol 9a, ketone 16 was subjected to enantioselective reduction ${ }^{13,14)}$ by using chiral prolinol ligand $(R)-\mathbf{1 7}$ or $(S)-\mathbf{1 7}$, followed by reduction of a nitro group to give optically pure aniline $(S)-\mathbf{1 0 a}$ and $(R)-\mathbf{1 0 a}$, respectively. Optically pure and stereochemically inversed bisamidines $(R)-\mathbf{1 3} \mathbf{j}$ and $(S)$-13j were synthesized from $(S)$ and $(R)-\mathbf{1 0 a}$ by the same method as their racemate, respectively.

The syntheses of 5-amidino-2-hydroxycinnamyl derivatives $(\mathbf{2 2} \mathbf{a}-\mathbf{c}, \mathbf{2 2} \mathbf{f}-\mathbf{h})$ are outlined in Chart 6. After treatment of compound $\mathbf{1 8}$ with ylide, a hydroxyl group of resulting compound 19 was protected to give $O$-protected cinnamaldehyde 20. Compound $\mathbf{2 0}$ was converted to cyclized compounds (21a, 21c, 21d, 21e) by a similar method described in Chart 2. A methoxymethyl group of 21a was converted to a methyl group by 3 steps to give compound $\mathbf{2 1 b}$. Cyclized compounds 21a-e were converted to desired compounds 22a-e by a similar method described in Chart 2 . Furthermore, carboxylic acids $\mathbf{2 2 f}-\mathbf{h}$ were synthesized from the corresponding esters $22 \mathbf{c}$ - e by acid hydrolysis.

\section{Results and Discussion}

In vitro $\mathrm{FXa}$ and trypsin inhibitory activities of all compounds were evaluated and expressed as $\mathrm{IC}_{50}$ values.

As described above, we considered that it is important not
Table 1. FXa and Trypsin Inhibitory Activities of Cinnamyl Compound 13a and Naphthyl Compound 1<smiles>CCOS(=O)(=O)N1c2ccc(OC3CCN(C(C)=N)CC3)cc2CC1[Hg]C(=N)N</smiles>

\begin{tabular}{|c|c|c|c|c|}
\hline \multirow{2}{*}{ Compd. ${ }^{a}{ }^{2}$} & \multirow{2}{*}{$\mathrm{L}$} & \multicolumn{2}{|c|}{$\mathrm{IC}_{50}(\mathrm{nM})$} & \multirow{2}{*}{ Ratio $^{b)}$} \\
\hline & & FXa & Trypsin & \\
\hline $13 a$ & & 12 & 120 & 10 \\
\hline 1 & & 11 & 52 & 4.7 \\
\hline
\end{tabular}

a) All compounds were synthesized and evaluated as their hydrochlorides. b) Ratio: the $\mathrm{IC}_{50}$ values for Trypsin $v s$. FXa (Trypsin/FXa).

only to enhance FXa inhibitory activity but to improve selectivity over trypsin. From this viewpoint, we reexamined various moieties of the indoline compound we have reported previously. $^{7,8)}$

First, we focused on the naphthalene structure attached to an indoline ring (Table 1). We synthesized a cinnamyl compound 13a, a ring-opening form of naphthalene, and tested its inhibitory activity against FXa and trypsin. Compound 13a exhibited potent FXa inhibitory activity even equal to naphthyl compound 1. Moreover, 13a exhibited 10-fold enhanced selectivity for FXa over trypsin. This selectivity was superior to that of naphthyl compound 1. According to this result, cinnamyl moiety seemed to be appropriate as an alter- 
Table 2. FXa and Trypsin Inhibitory Activities of Compounds 13a- e<smiles>CCOS(=O)(=O)N1c2ccc(OC3CCN(C(C)=N)CC3)cc2CC1[IH]c1cccc(C(=N)N)c1</smiles>

\begin{tabular}{|c|c|c|c|c|}
\hline \multirow{2}{*}{ Compd. ${ }^{a)}$} & \multirow{2}{*}{$\mathrm{L}$} & \multicolumn{2}{|c|}{$\mathrm{IC}_{50}(\mathrm{nM})$} & \multirow{2}{*}{ Ratio $^{b)}$} \\
\hline & & $\mathrm{FXa}$ & Trypsin & \\
\hline $13 a$ & & 12 & 120 & 10 \\
\hline $13 b$ & & 21 & 160 & 7.6 \\
\hline $13 c$ & & 24 & 140 & 5.8 \\
\hline $13 d$ & & 24 & 180 & 7.5 \\
\hline $13 \mathrm{e}$ & & 49 & 130 & 2.7 \\
\hline
\end{tabular}

a) All compounds were synthesized and evaluated as their hydrochlorides. b) Ratio: the $\mathrm{IC}_{50}$ values for Trypsin $v$. FXa (Trypsin/FXa).

Table 3. FXa and Trypsin Inhibitory Activities of Compounds 13a, 13f, 13g, 13i and 13j

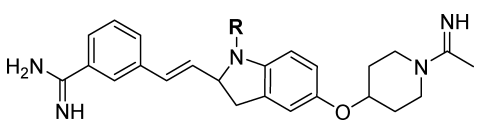

\begin{tabular}{|c|c|c|c|c|}
\hline \multirow{2}{*}{ Compd. $^{a)}$} & \multirow{2}{*}{$\mathrm{R}$} & \multicolumn{2}{|c|}{$\mathrm{IC}_{50}(\mathrm{nM})$} & \multirow{2}{*}{ Ratio $^{b}$} \\
\hline & & $\mathrm{FXa}$ & Trypsin & \\
\hline $13 f$ & $\mathrm{SO}_{2} \mathrm{CH}_{2} \mathrm{CO}_{2} \mathrm{Et}$ & 11 & 110 & 10 \\
\hline $13 \mathbf{j}$ & $\mathrm{SO}_{2} \mathrm{CH}_{2} \mathrm{CO}_{2} \mathrm{H}$ & 9.4 & 160 & 17 \\
\hline $13 \mathrm{~g}$ & $\mathrm{COCH}_{3}$ & 17 & 190 & 11 \\
\hline $13 \mathbf{i}$ & $\mathrm{COCH}_{2} \mathrm{OH}$ & 11 & 65 & 5.9 \\
\hline 13a & $\mathrm{SO}_{2} \mathrm{Et}$ & 12 & 120 & 10 \\
\hline
\end{tabular}

a) All compounds were synthesized and evaluated as their hydrochlorides. b) Ratio: the $\mathrm{IC}_{50}$ values for Trypsin $v$. FXa (Trypsin/FXa).

native to naphthyl moiety.

To optimize this moiety, substituents were introduced on the double bond and their effects were evaluated (Table 2). An introduction of a methyl or an ethyl group on the $\beta$-position of the cinnamyl moiety $(\mathbf{1 3 b}, \mathbf{1 3 c})$ resulted in a decline of FXa inhibitory activity and selectivity compared to that of 13a. To examine the effect of an introduction of a polar substituent at this position, methoxymethylene compound 13d was synthesized and its inhibitory activity was evaluated. However, the activity was similar as those of $\mathbf{1 3 b}$ and $\mathbf{1 3 c}$. On the other hand, compound 13e, having a methyl group on the $\alpha$-position, exhibited much less inhibitory activity and selectivity. According to these results, $\alpha, \beta$-non-substituted cinnamyl moiety seems to be more suitable.

Next, the effect of the substitution of the nitrogen atom of the indoline ring was examined (Table 3). Conversion of ethanesulfonyl moiety of 13a into sulfonylacetic acid (13j) and its ester form (13f) brought about similar FXa inhibitory activities as 13a, but as for $\mathbf{1 3} \mathbf{j}$, selectivity over trypsin was
Table 4. FXa and Trypsin Inhibitory Activities of Compounds 13f, 13k$\mathbf{r}, \mathbf{1 3 t}$ and $13 \mathbf{u}$

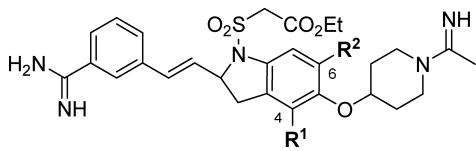

\begin{tabular}{|c|c|c|c|c|c|}
\hline \multirow{2}{*}{ Compd ${ }^{a}{ }^{\prime}$} & \multirow{2}{*}{$\mathrm{R}^{1}$} & \multirow{2}{*}{$\mathrm{R}^{2}$} & \multicolumn{2}{|c|}{$\mathrm{IC}_{50}(\mathrm{nM})$} & \multirow{2}{*}{ Ratio $^{b)}$} \\
\hline & & & FXa & Trypsin & \\
\hline $13 k$ & $\mathrm{H}$ & $\mathrm{Cl}$ & 14 & 39 & 2.8 \\
\hline $13 \mathrm{~m}$ & $\mathrm{H}$ & $\mathrm{F}$ & 11 & 160 & 15 \\
\hline 130 & $\mathrm{H}$ & $\mathrm{CF}_{3}$ & 6.2 & 20 & 3.2 \\
\hline $13 q$ & $\mathrm{H}$ & $\mathrm{Me}$ & 13 & 84 & 6.5 \\
\hline $13 \mathrm{t}$ & $\mathrm{H}$ & $\mathrm{CO}_{2} \mathrm{Et}$ & 14 & 64 & 4.6 \\
\hline $13 u$ & $\mathrm{H}$ & $\mathrm{CONH}_{2}$ & 8.7 & 25 & 2.9 \\
\hline $13 I$ & $\mathrm{Cl}$ & $\mathrm{H}$ & 9.5 & 200 & 21 \\
\hline $13 n$ & $\mathrm{~F}$ & $\mathrm{H}$ & 11 & 160 & 15 \\
\hline $13 p$ & $\mathrm{CF}_{3}$ & $\mathrm{H}$ & 7.3 & 290 & 40 \\
\hline $13 r$ & $\mathrm{Me}$ & $\mathrm{H}$ & 8.1 & 220 & 27 \\
\hline $13 \mathrm{f}$ & $\mathrm{H}$ & $\mathrm{H}$ & 11 & 110 & 10 \\
\hline
\end{tabular}

a) All compounds were synthesized and evaluated as their hydrochlorides. $b$ ) Ratio: the $\mathrm{IC}_{50}$ values for Trypsin vs. FXa (Trypsin/FXa).

improved. On the other hand, acetyl compound 13g showed slightly less FXa inhibitory activity. An introduction of a hydroxyl group on the acetyl moiety (13i) resulted in similar FXa inhibitory activity as 13a. However, 13i also exhibited potent inhibitory activity against trypsin. These results were also confirmed for the naphthylindoline derivatives, ${ }^{8)}$ and suggest that sulfonylacetic acid type structures are favorable at this position.

Furthermore, the effects of the substituents on the indoline ring (4- vs. 6-position) were examined (Table 4). Compounds having a fluorine atom, a bioisoster of a hydrogen atom, on the 4- $(\mathbf{1 3 n})$ or 6-position $(\mathbf{1 3 m})$ resulted in the same result as non-substituted compound $\mathbf{1 3 f}$. Compounds having a more bulky group than a hydrogen atom $(\mathbf{1 3 k}, \mathbf{1 3 0}, \mathbf{1 3 q}, \mathbf{1 3 t}, \mathbf{1 3 \mathbf { u }})$ at the 6-position exhibited potent inhibitory activities against both FXa and trypsin. On the other hand, compounds (13I, 13p, 13r) having a similar bulky group as above at the 4-position exhibited potent FXa inhibitory activity. Moreover, selectivity over trypsin was improved compared to that of $\mathbf{1 3 f}$. These results suggest that an introduction at the 4- or 6-position does not have an influence against FXa inhibitory activity, but they do have a significant influence against trypsin inhibitory activity. Furthermore, the bulkiness was important for these activities, whereas the electron property (electron withdrawing/donating group) was not so important. From these results, it seemed that the 4-substitution is preferable to 6-substitution.

All compounds discussed above are racemates having an asymmetric carbon atom at the 2-position of the indoline ring. We previously reported that the $(R)$-naphthylindoline derivative exhibited over 20 times higher FXa inhibitory activity than the corresponding $(S)$-isomer. ${ }^{7)}$ Based on this result, each of the enantiomers $((R)$ - and $(S)$-13j) of a cinnamylindoline derivative 13j was prepared and their FXa and trypsin inhibitory activities were evaluated (Table 5). However, to our surprise, the FXa inhibitory activities of $(R)$ - and $(S)$-13j were nearly equal. We thought that this result was caused by the structural flexibility of cinnnamyl moiety. The 
Table 5. FXa and Trypsin Inhibitory Activities of Compounds $(R)-\mathbf{1 3} \mathbf{j}$, $(S)-\mathbf{1 3} \mathbf{j}$ and $\mathbf{1 3} \mathbf{j}$

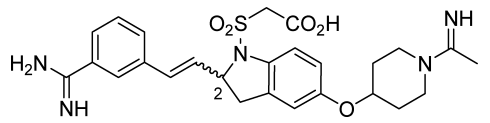

\begin{tabular}{ccccc}
\hline \hline \multirow{2}{*}{ Compd. ${ }^{a)}$} & & \multicolumn{2}{c}{$\mathrm{IC}_{50}(\mathrm{nM})$} & \\
\cline { 3 - 4 } & 2-position & Ratio & \\
& & $\mathrm{FXa}$ & Trypsin & \\
\hline$(R)-\mathbf{1 3} \mathbf{j}$ & $R$ & 11 & 130 & 12 \\
$(S)-\mathbf{1 3} \mathbf{j}$ & $S$ & 16 & 320 & 20 \\
$\mathbf{1 3 j}$ & $R S$ & 9.4 & 160 & 17 \\
& & &
\end{tabular}

a) All compounds were synthesized and evaluated as their hydrochlorides. b) Ratio: the $\mathrm{IC}_{50}$ values for Trypsin $v$. FXa (Trypsin/FXa).

Table 6. FXa and Trypsin Inhibitory Activities of Compounds $22 \mathbf{a}-\mathbf{c}$, $\mathbf{2 2 f}-\mathbf{h}, \mathbf{1 3} \mathbf{a}$ and $\mathbf{1 3} \mathbf{j}$

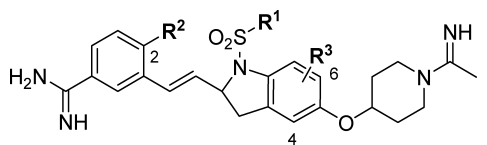

\begin{tabular}{|c|c|c|c|c|c|c|}
\hline \multirow{2}{*}{ Compd. $\left.{ }^{a}\right)$} & \multirow{2}{*}{$\mathrm{R}^{1}$} & \multirow{2}{*}{$\mathrm{R}^{2}$} & \multirow{2}{*}{$\mathrm{R}^{3}$} & \multicolumn{2}{|c|}{$\mathrm{IC}_{50}(\mathrm{nM})$} & \multirow{2}{*}{ Ratio $^{b)}$} \\
\hline & & & & $\mathrm{FXa}$ & Trypsin & \\
\hline $22 a$ & Et & $\mathrm{OH}$ & $\mathrm{H}$ & 7.0 & 1300 & 190 \\
\hline $22 b$ & Et & $\mathrm{OMe}$ & $\mathrm{H}$ & 280 & 2000 & 7.1 \\
\hline $22 \mathrm{c}$ & $\mathrm{CH}_{2} \mathrm{CO}_{2} \mathrm{Et}$ & $\mathrm{OH}$ & $\mathrm{H}$ & 6.8 & 1900 & 280 \\
\hline $22 f$ & $\mathrm{CH}_{2} \mathrm{CO}_{2} \mathrm{H}$ & $\mathrm{OH}$ & $\mathrm{H}$ & 4.4 & 1500 & 340 \\
\hline $22 \mathrm{~g}$ & $\mathrm{CH}_{2} \mathrm{CO}_{2} \mathrm{H}$ & $\mathrm{OH}$ & $4-\mathrm{Cl}$ & 20 & 2600 & 130 \\
\hline $22 \mathrm{~h}$ & $\mathrm{CH}_{2} \mathrm{CO}_{2} \mathrm{H}$ & $\mathrm{OH}$ & $6-\mathrm{Cl}$ & 11 & 1200 & 110 \\
\hline $13 \mathbf{a}$ & Et & $\mathrm{H}$ & $\mathrm{H}$ & 12 & 120 & 10 \\
\hline $\mathbf{1 3 j}$ & $\mathrm{CH}_{2} \mathrm{CO}_{2} \mathrm{H}$ & $\mathrm{H}$ & $\mathrm{H}$ & 9.4 & 160 & 17 \\
\hline
\end{tabular}

a) All compounds were synthesized and evaluated as their hydrochlorides. b) Ratio: the $\mathrm{IC}_{50}$ values for Trypsin $v$. FXa (Trypsin/FXa).

structure of the cinnamyl group was less rigid than that of the naphthyl group, so it seemed that the difference of FXa inhibitory activity between $(R)$ - and $(S)$-cinnamylindoline derivative was not observed. Moreover, from the synthetic aspect, selective synthesis of the one enantiomer needs more effort compared to that of its racemate. According to this result, we considered that it is suitable to develop our cinnamylindoline derivatives as a racemate.

The effect of the substituents on the benzene ring of the cinnamyl moiety was examined (Table 6). Compound 22a, having a hydroxyl group at the 2-position, exhibited more potent FXa inhibitory activity and much higher selectivity over trypsin than non-substituted compound 13a. However, compound $\mathbf{2 2 b}$, having a methoxy group on the same position, showed much lower inhibitory activity. This result suggests that the hydroxyl group on this position is highly important for both FXa inhibitory activity and selectivity over trypsin. From the X-ray crystallographic analysis, FXa has an amino acid residue Ser 195 in its S1 pocket ${ }^{7,15}$ and Ser 195 is located nearby the benzamidine moieties of FXa inhibitors. We speculate that the hydroxyl group at the 2-position of the benzene ring makes a hydrogen bond with Ser 195, whereas it makes a water-mediated hydrogen bond, a relatively indirect interaction, with Ser 195 in the S1 pocket of trypsin. ${ }^{16-19)}$ It seems that this difference leads to both po-
Table 7. FXa and Other Serine Protease Inhibitory Activities of Compound 22f

\begin{tabular}{lc}
\hline \hline Enzyme & $\mathrm{IC}_{50}(\mathrm{nM})$ \\
\hline FXa & 4.4 \\
Trypsin & 1500 \\
FIIa & $>100000$ \\
Plasmin & 16000 \\
\hline
\end{tabular}

Table 8. In Vitro Anticoagulant Activities of Compounds $22 \mathbf{f}$ and $(R)-\mathbf{2}$

\begin{tabular}{|c|c|c|}
\hline \multirow{2}{*}{ Compd. } & \multicolumn{2}{|c|}{ Human $\mathrm{CT}_{2}(\mu \mathrm{M})^{a)}$} \\
\hline & PT & APTT \\
\hline $22 f$ & 0.39 & 0.34 \\
\hline$(R)-\mathbf{2}$ & 0.48 & 1.2 \\
\hline
\end{tabular}

a) The concentration required to double clotting time.

tent FXa inhibitory activity and the enzyme selectivity. On the other hand, the methoxy group of $\mathbf{2 2 b}$ makes it difficult to interact with the S1 pocket of both FXa and trypsin, so this caused a significant decline of inhibitory activity. Furthermore, the replacement of the ethanesulfonyl group by a carboxymethylsulfonyl group (and its ester form) gave the most potent FXa inhibitory activity (22a vs. 22f and 22c). Moreover, selectivity over trypsin of $\mathbf{2 2 f}$ was over 20 times improved compared to that of $\mathbf{1 3} \mathbf{j}$. However, $\mathbf{2 2} \mathrm{g}$ and $\mathbf{2 2} \mathbf{h}$ with a chlorine atom at the 4- or 6-positon of the indoline ring exhibited, despite the result shown in Table 4, lower FXa inhibitory activities than that of non-substituted compound 22f.

Compound 22f also exhibited good enzyme selectivity over other serine proteases (Table 7). Therefore, compound 22f had the best profile in both the FXa inhibitory activity and enzyme selectivity among all compounds we have synthesized and tested.

We evaluated the anticoagulant activity of $\mathbf{2 2 f}$ on both prothrombin time (PT) and activated partial thromboplastin time (APTT) in human plasma (Table 8). $\mathrm{CT}_{2}$ values, the concentration required to achieve $200 \%$ relative clotting time, of $\mathbf{2 2 f}$ were $0.39 \mu \mathrm{M}(\mathrm{PT})$ and $0.34 \mu \mathrm{M}$ (APTT), whereas those of naphthyl compound $(R)-2$ were $0.48 \mu \mathrm{M}(\mathrm{PT})$ and $1.2 \mu \mathrm{M}$ (APTT), respectively. These results indicate that compound 22f has more potent in vitro anticoagulant activity than $(R)-\mathbf{2}$.

In conclusion, we synthesized many cinnamylindoline derivatives to find compounds having potent FXa inhibitory activities and selectivity over trypsin. As a result, we found that some novel derivatives having 2-hydroxycinnnamyl moieties showed high FXa inhibitory activities and selectivity over trypsin. Among them, $(E)-2-\{5-[1-($ acetimidoyl)piperidin-4yloxy]-2-[2-(5-amidino-2-hydroxyphenyl)ethen-1-yl]indolin1-ylsulfonyl \}acetic acid (22f) exhibited potent FXa inhibitory activity and good enzyme selectivity in vitro. Furthermore, compound $22 \mathrm{f}$ exhibited potent in vitro anticoagulant activity in human plasma, although oral anticoagulant activity remains to be determined. Our further synthetic efforts are in progress and the results will be disclosed in the next report. 
Table 9. Elemental Analysis Data for Cinnamylindoline Derivatives $\mathbf{1 3}$ and 22

\begin{tabular}{|c|c|c|c|c|c|c|c|}
\hline \multirow{2}{*}{ Compd. } & \multirow{2}{*}{ Formula } & \multicolumn{6}{|c|}{ Analysis (\%) Calcd (Found) } \\
\hline & & $\mathrm{C}$ & $\mathrm{H}$ & $\mathrm{N}$ & $\mathrm{Cl}$ & $\mathrm{S}$ & $\mathrm{F}$ \\
\hline $13 \mathbf{a}$ & $\mathrm{C}_{26} \mathrm{H}_{33} \mathrm{~N}_{5} \mathrm{O}_{3} \mathrm{~S} \cdot 2.0 \mathrm{HCl} \cdot 2.8 \mathrm{H}_{2} \mathrm{O}$ & $\begin{array}{c}50.45 \\
(50.13)\end{array}$ & $\begin{array}{c}6.61 \\
(6.65)\end{array}$ & $\begin{array}{c}11.31 \\
(11.65)\end{array}$ & $\begin{array}{c}11.45 \\
(11.52\end{array}$ & $\begin{array}{c}5.18 \\
(5.22)\end{array}$ & - \\
\hline $13 b$ & $\mathrm{C}_{27} \mathrm{H}_{35} \mathrm{~N}_{5} \mathrm{O}_{3} \mathrm{~S} \cdot 2.4 \mathrm{HCl} \cdot 1.9 \mathrm{H}_{2} \mathrm{O}$ & $\begin{array}{c}51.36 \\
(51.62)\end{array}$ & $\begin{array}{c}6.58 \\
(6.93)\end{array}$ & $\begin{array}{c}11.09 \\
(11.20)\end{array}$ & $\begin{array}{c}13.48 \\
(13.12)\end{array}$ & $\begin{array}{c}5.08 \\
(5.02)\end{array}$ & - \\
\hline $13 c$ & $\mathrm{C}_{28} \mathrm{H}_{37} \mathrm{~N}_{5} \mathrm{O}_{3} \mathrm{~S} \cdot 2.1 \mathrm{HCl} \cdot 1.8 \mathrm{H}_{2} \mathrm{O}$ & $\begin{array}{c}53.16 \\
(52.98)\end{array}$ & $\begin{array}{c}6.80 \\
(7.10)\end{array}$ & $\begin{array}{c}11.07 \\
(11.12)\end{array}$ & $\begin{array}{c}11.77 \\
(11.48)\end{array}$ & $\begin{array}{c}5.07 \\
(5.36)\end{array}$ & - \\
\hline 13d & $\mathrm{C}_{28} \mathrm{H}_{37} \mathrm{~N}_{5} \mathrm{O}_{4} \mathrm{~S} \cdot 2.0 \mathrm{HCl} \cdot 2.9 \mathrm{H}_{2} \mathrm{O}$ & $\begin{array}{c}50.58 \\
(50.36)\end{array}$ & $\begin{array}{c}6.79 \\
(6.42)\end{array}$ & $\begin{array}{c}10.53 \\
(10.55)\end{array}$ & $\begin{array}{c}10.66 \\
(10.66)\end{array}$ & $\begin{array}{c}4.82 \\
(4.91)\end{array}$ & - \\
\hline $13 e$ & $\mathrm{C}_{27} \mathrm{H}_{35} \mathrm{~N}_{5} \mathrm{O}_{3} \mathrm{~S} \cdot 2.0 \mathrm{HCl} \cdot 2.5 \mathrm{H}_{2} \mathrm{O}$ & $\begin{array}{c}51.67 \\
(51.78)\end{array}$ & $\begin{array}{c}6.75 \\
(6.47)\end{array}$ & $\begin{array}{c}11.16 \\
(11.23)\end{array}$ & $\begin{array}{c}11.30 \\
(11.12)\end{array}$ & $\begin{array}{c}5.11 \\
(5.28)\end{array}$ & - \\
\hline $13 f$ & $\mathrm{C}_{28} \mathrm{H}_{35} \mathrm{~N}_{5} \mathrm{O}_{5} \mathrm{~S} \cdot 2.0 \mathrm{HCl} \cdot 2.5 \mathrm{H}_{2} \mathrm{O}$ & $\begin{array}{c}50.07 \\
(50.16)\end{array}$ & $\begin{array}{c}6.30 \\
(5.95)\end{array}$ & $\begin{array}{c}10.43 \\
(10.53)\end{array}$ & $\begin{array}{c}10.56 \\
(10.46)\end{array}$ & $\begin{array}{c}4.77 \\
(4.66)\end{array}$ & - \\
\hline $13 g$ & $\mathrm{C}_{26} \mathrm{H}_{31} \mathrm{~N}_{5} \mathrm{O}_{2} \cdot 2.1 \mathrm{HCl} \cdot 2.5 \mathrm{H}_{2} \mathrm{O}$ & $\begin{array}{c}55.06 \\
(55.42)\end{array}$ & $\begin{array}{c}6.77 \\
(6.75)\end{array}$ & $\begin{array}{c}12.35 \\
(11.97)\end{array}$ & $\begin{array}{c}13.13 \\
(12.99)\end{array}$ & - & - \\
\hline $13 \mathbf{i}$ & $\mathrm{C}_{26} \mathrm{H}_{31} \mathrm{~N}_{5} \mathrm{O}_{3} \cdot 2.0 \mathrm{HCl} \cdot 2.7 \mathrm{H}_{2} \mathrm{O}$ & $\begin{array}{c}53.55 \\
(53.34)\end{array}$ & $\begin{array}{c}6.64 \\
(6.38)\end{array}$ & $\begin{array}{c}12.01 \\
(12.08)\end{array}$ & $\begin{array}{c}12.16 \\
(12.39)\end{array}$ & - & - \\
\hline $13 \mathbf{j}$ & $\mathrm{C}_{26} \mathrm{H}_{31} \mathrm{~N}_{5} \mathrm{O}_{5} \mathrm{~S} \cdot 2.4 \mathrm{HCl} \cdot 1.7 \mathrm{H}_{2} \mathrm{O}$ & $\begin{array}{c}48.51 \\
(48.77)\end{array}$ & $\begin{array}{c}5.76 \\
(5.64)\end{array}$ & $\begin{array}{c}10.88 \\
(10.62)\end{array}$ & $\begin{array}{c}13.22 \\
(13.10)\end{array}$ & $\begin{array}{c}4.98 \\
(4.95)\end{array}$ & - \\
\hline $13 \mathbf{k}$ & $\mathrm{C}_{28} \mathrm{H}_{34} \mathrm{ClN}_{5} \mathrm{O}_{5} \mathrm{~S} \cdot 1.9 \mathrm{HCl} \cdot 2.3 \mathrm{H}_{2} \mathrm{O}$ & $\begin{array}{c}48.12 \\
(48.01)\end{array}$ & $\begin{array}{c}5.84 \\
(5.67)\end{array}$ & $\begin{array}{c}10.02 \\
(10.07)\end{array}$ & $\begin{array}{c}14.71 \\
(14.70)\end{array}$ & $\begin{array}{c}4.59 \\
(4.80)\end{array}$ & - \\
\hline 131 & $\mathrm{C}_{28} \mathrm{H}_{34} \mathrm{ClN}_{5} \mathrm{O}_{5} \mathrm{~S} \cdot 2.1 \mathrm{HCl} \cdot 1.6 \mathrm{H}_{2} \mathrm{O}$ & $\begin{array}{c}48.49 \\
(48.28)\end{array}$ & $\begin{array}{c}5.71 \\
(5.99)\end{array}$ & $\begin{array}{c}10.10 \\
(10.22)\end{array}$ & $\begin{array}{c}15.85 \\
(15.83)\end{array}$ & $\begin{array}{c}4.62 \\
(4.80)\end{array}$ & - \\
\hline $13 \mathrm{~m}$ & $\mathrm{C}_{28} \mathrm{H}_{34} \mathrm{FN}_{5} \mathrm{O}_{5} \mathrm{~S} \cdot 2.1 \mathrm{HCl} \cdot 1.4 \mathrm{H}_{2} \mathrm{O}$ & $\begin{array}{c}49.94 \\
(49.91)\end{array}$ & $\begin{array}{c}5.82 \\
(5.95)\end{array}$ & $\begin{array}{c}10.40 \\
(10.52)\end{array}$ & $\begin{array}{c}11.05 \\
(11.16)\end{array}$ & $\begin{array}{c}4.76 \\
(4.66)\end{array}$ & $\begin{array}{l}2.82 \\
(2.67)\end{array}$ \\
\hline $13 n$ & $\mathrm{C}_{28} \mathrm{H}_{34} \mathrm{FN}_{5} \mathrm{O}_{5} \mathrm{~S} \cdot 1.7 \mathrm{HCl} \cdot 2.5 \mathrm{H}_{2} \mathrm{O}$ & $\begin{array}{c}49.55 \\
(49.63)\end{array}$ & $\begin{array}{c}6.04 \\
(5.92)\end{array}$ & $\begin{array}{c}10.32 \\
(10.46)\end{array}$ & $\begin{array}{c}8.88 \\
(8.73)\end{array}$ & $\begin{array}{c}4.72 \\
(4.61)\end{array}$ & $\begin{array}{c}2.80 \\
(2.58)\end{array}$ \\
\hline 130 & $\mathrm{C}_{29} \mathrm{H}_{34} \mathrm{~F}_{3} \mathrm{~N}_{5} \mathrm{O}_{5} \mathrm{~S} \cdot 2.1 \mathrm{HCl} \cdot 2.2 \mathrm{H}_{2} \mathrm{O}$ & $\begin{array}{c}47.21 \\
(47.32)\end{array}$ & $\begin{array}{c}5.53 \\
(5.28)\end{array}$ & $\begin{array}{c}9.49 \\
(9.61)\end{array}$ & $\begin{array}{c}10.09 \\
(10.01)\end{array}$ & $\begin{array}{c}4.35 \\
(4.19)\end{array}$ & $\begin{array}{c}7.72 \\
(7.76)\end{array}$ \\
\hline $13 p$ & $\mathrm{C}_{29} \mathrm{H}_{34} \mathrm{~F}_{3} \mathrm{~N}_{5} \mathrm{O}_{5} \mathrm{~S} \cdot 2.3 \mathrm{HCl} \cdot 1.6 \mathrm{H}_{2} \mathrm{O}$ & $\begin{array}{c}47.43 \\
(47.60)\end{array}$ & $\begin{array}{c}5.42 \\
(5.35)\end{array}$ & $\begin{array}{c}9.54 \\
(9.41)\end{array}$ & $\begin{array}{c}11.10 \\
(11.22)\end{array}$ & $\begin{array}{c}4.37 \\
(4.25)\end{array}$ & $\begin{array}{c}7.76 \\
(7.76)\end{array}$ \\
\hline $13 q$ & $\mathrm{C}_{29} \mathrm{H}_{37} \mathrm{~N}_{5} \mathrm{O}_{5} \mathrm{~S} \cdot 2.1 \mathrm{HCl} \cdot 1.3 \mathrm{H}_{2} \mathrm{O}$ & $\begin{array}{c}52.17 \\
(51.91)\end{array}$ & $\begin{array}{c}6.30 \\
(6.09)\end{array}$ & $\begin{array}{c}10.49 \\
(10.73)\end{array}$ & $\begin{array}{c}11.15 \\
(11.44)\end{array}$ & $\begin{array}{c}4.80 \\
(4.70)\end{array}$ & - \\
\hline $13 r$ & $\mathrm{C}_{29} \mathrm{H}_{37} \mathrm{~N}_{5} \mathrm{O}_{5} \mathrm{~S} \cdot 2.1 \mathrm{HCl} \cdot 1.3 \mathrm{H}_{2} \mathrm{O}$ & $\begin{array}{c}52.17 \\
(52.24)\end{array}$ & $\begin{array}{c}6.30 \\
(6.00)\end{array}$ & $\begin{array}{c}10.49 \\
(10.69)\end{array}$ & $\begin{array}{c}11.15 \\
(11.15)\end{array}$ & $\begin{array}{c}4.80 \\
(4.81)\end{array}$ & - \\
\hline $13 t$ & $\mathrm{C}_{31} \mathrm{H}_{39} \mathrm{~N}_{5} \mathrm{O}_{7} \mathrm{~S} \cdot 2.0 \mathrm{HCl} \cdot 2.1 \mathrm{H}_{2} \mathrm{O}$ & $\begin{array}{c}50.56 \\
(50.75)\end{array}$ & $\begin{array}{c}6.19 \\
(5.93)\end{array}$ & $\begin{array}{c}9.51 \\
(9.51)\end{array}$ & $\begin{array}{c}9.63 \\
(9.55)\end{array}$ & $\begin{array}{c}4.35 \\
(4.36)\end{array}$ & - \\
\hline $13 u$ & $\mathrm{C}_{29} \mathrm{H}_{36} \mathrm{~N}_{6} \mathrm{O}_{6} \mathrm{~S} \cdot 2.4 \mathrm{HCl} \cdot 2.5 \mathrm{H}_{2} \mathrm{O}$ & $\begin{array}{c}47.76 \\
(47.63)\end{array}$ & $\begin{array}{c}6.00 \\
(5.76)\end{array}$ & $\begin{array}{c}11.52 \\
(11.67)\end{array}$ & $\begin{array}{c}11.67 \\
(11.68)\end{array}$ & $\begin{array}{c}4.40 \\
(4.64)\end{array}$ & - \\
\hline $22 a$ & $\mathrm{C}_{26} \mathrm{H}_{33} \mathrm{~N}_{5} \mathrm{O}_{4} \mathrm{~S} \cdot 2.0 \mathrm{HCl} \cdot 2.6 \mathrm{H}_{2} \mathrm{O}$ & $\begin{array}{c}49.46 \\
(49.77)\end{array}$ & $\begin{array}{c}6.42 \\
(6.13)\end{array}$ & $\begin{array}{c}11.09 \\
(11.23)\end{array}$ & $\begin{array}{c}11.23 \\
(11.02)\end{array}$ & $\begin{array}{c}5.08 \\
(4.81)\end{array}$ & - \\
\hline $22 b$ & $\mathrm{C}_{27} \mathrm{H}_{35} \mathrm{~N}_{5} \mathrm{O}_{4} \mathrm{~S} \cdot 2.1 \mathrm{HCl} \cdot 1.6 \mathrm{H}_{2} \mathrm{O}$ & $\begin{array}{c}51.39 \\
(51.32)\end{array}$ & $\begin{array}{c}6.44 \\
(6.47)\end{array}$ & $\begin{array}{c}11.10 \\
(10.96)\end{array}$ & $\begin{array}{c}11.80 \\
(11.89)\end{array}$ & $\begin{array}{c}5.08 \\
(5.27)\end{array}$ & - \\
\hline $22 c$ & $\mathrm{C}_{28} \mathrm{H}_{35} \mathrm{~N}_{5} \mathrm{O}_{6} \mathrm{~S} \cdot 2.0 \mathrm{HCl} \cdot 1.2 \mathrm{H}_{2} \mathrm{O}$ & $\begin{array}{c}50.79 \\
(50.98)\end{array}$ & $\begin{array}{c}5.69 \\
(5.81)\end{array}$ & $\begin{array}{c}10.58 \\
(10.59)\end{array}$ & $\begin{array}{c}10.71 \\
(10.69)\end{array}$ & $\begin{array}{c}4.84 \\
(4.72)\end{array}$ & - \\
\hline $22 f$ & $\mathrm{C}_{26} \mathrm{H}_{31} \mathrm{~N}_{5} \mathrm{O}_{6} \mathrm{~S} \cdot 2.5 \mathrm{HCl} \cdot 0.5 \mathrm{H}_{2} \mathrm{O}$ & $\begin{array}{c}48.66 \\
(48.53)\end{array}$ & $\begin{array}{c}5.42 \\
(5.79)\end{array}$ & $\begin{array}{c}10.91 \\
(11.08)\end{array}$ & $\begin{array}{c}13.81 \\
(13.46)\end{array}$ & $\begin{array}{c}5.00 \\
(5.09)\end{array}$ & - \\
\hline $22 \mathrm{~g}$ & $\mathrm{C}_{26} \mathrm{H}_{30} \mathrm{ClN}_{5} \mathrm{O}_{6} \mathrm{~S} \cdot 1.9 \mathrm{HCl} \cdot 2.9 \mathrm{H}_{2} \mathrm{O}$ & $\begin{array}{c}44.77 \\
(44.94)\end{array}$ & $\begin{array}{c}5.45 \\
(5.19)\end{array}$ & $\begin{array}{c}10.04 \\
(10.11)\end{array}$ & $\begin{array}{c}14.74 \\
(14.90)\end{array}$ & $\begin{array}{c}4.60 \\
(4.33)\end{array}$ & - \\
\hline $22 \mathrm{~h}$ & $\mathrm{C}_{26} \mathrm{H}_{30} \mathrm{ClN}_{5} \mathrm{O}_{6} \mathrm{~S} \cdot 2.0 \mathrm{HCl} \cdot 2.1 \mathrm{H}_{2} \mathrm{O}$ & $\begin{array}{c}45.47 \\
(45.34)\end{array}$ & $\begin{array}{c}5.31 \\
(5.46)\end{array}$ & $\begin{array}{c}10.20 \\
(10.40)\end{array}$ & $\begin{array}{c}15.49 \\
(15.42)\end{array}$ & $\begin{array}{c}4.67 \\
(4.70)\end{array}$ & - \\
\hline
\end{tabular}

\section{Experimental}

${ }^{1} \mathrm{H}$-NMR spectra were obtained on a Varian Mercury 400 or Unity Inova 500 FT-NMR spectrometer and were reported as $\delta$ values relative to $\mathrm{Me}_{4} \mathrm{Si}$ as the internal standard. Abbreviations of the ${ }^{1} \mathrm{H}-\mathrm{NMR}$ peak patterns are as follows: $b s=$ broad singlet, $s=$ singlet, $d=$ doublet, $d d=$ double doublet, $\mathrm{t}=$ triplet, $\mathrm{dt}=$ double triplet, $\mathrm{q}=$ quartet and $\mathrm{m}=$ multiplet. Merck Silica gel $60(230-400$ mesh) was used in the column chromatography. Tetrahydrofuran, $N, N$-dimethylformamide, $N, N$-dimethylacetamide, and dimethylsulfoxide are abbreviated as THF, DMF, DMA and DMSO, respectively.

(E)-3-Cyanocinnamaldehyde (4a) To a solution of 3-cyanobenzaldehyde $3(4.50 \mathrm{~g}, 34.3 \mathrm{mmol})$ in toluene $(200 \mathrm{ml})$ was added (triphenylphosphoranylidene acetaldehyde $(13.6 \mathrm{~g}, 44.7 \mathrm{mmol})$ and the mixture was stirred at $70^{\circ} \mathrm{C}$ for $4 \mathrm{~h}$. The mixture was concentrated and the resulting residue was chromatographed on a silica gel column $\left(\mathrm{CH}_{2} \mathrm{Cl}_{2}\right)$ and then recrystallized (toluene/hexane) to give $4 \mathrm{a}(3.09 \mathrm{~g}, 19.7 \mathrm{mmol}, 57 \%)$ as pale yellow needles. ${ }^{1} \mathrm{H}-\mathrm{NMR}\left(\mathrm{CDCl}_{3}\right) \quad \delta: 6.76(1 \mathrm{H}, \quad \mathrm{dd}, J=7.4,16.1 \mathrm{~Hz}), 7.46 \quad(1 \mathrm{H}, \quad \mathrm{d}$,
$J=16.1 \mathrm{~Hz}), 7.58(1 \mathrm{H}, \mathrm{t}, J=7.8 \mathrm{~Hz}), 7.73(1 \mathrm{H}, \mathrm{d}, J=7.8 \mathrm{~Hz}), 7.81(1 \mathrm{H}, \mathrm{d}$, $J=7.8 \mathrm{~Hz}), 7.84(1 \mathrm{H}, \mathrm{s}), 9.76(1 \mathrm{H}, \mathrm{d}, J=7.4 \mathrm{~Hz})$.

Other derivatives $(\mathbf{4 b}, \mathbf{4 c})$ were similarly prepared.

4b: ${ }^{1} \mathrm{H}-\mathrm{NMR}\left(\mathrm{CDCl}_{3}\right) \delta: 2.07(3 \mathrm{H}, \mathrm{s}), 7.25(1 \mathrm{H}, \mathrm{s}), 7.59(1 \mathrm{H}, \mathrm{t}$, $J=7.8 \mathrm{~Hz}), 7.69(1 \mathrm{H}, \mathrm{d}, J=7.8 \mathrm{~Hz}), 7.74(1 \mathrm{H}, \mathrm{d}, J=7.8 \mathrm{~Hz}), 7.79(1 \mathrm{H}, \mathrm{s})$ $9.63(1 \mathrm{H}, \mathrm{s})$.

4c: ${ }^{1} \mathrm{H}-\mathrm{NMR}\left(\mathrm{CDCl}_{3}\right) \delta: 1.15(3 \mathrm{H}, \mathrm{t}, J=7.5 \mathrm{~Hz}), 2.52(2 \mathrm{H}, \mathrm{q}, J=7.5 \mathrm{~Hz})$, $7.19(1 \mathrm{H}, \mathrm{s}), 7.59(1 \mathrm{H}, \mathrm{t}, J=7.8 \mathrm{~Hz}), 7.68-7.73(2 \mathrm{H}, \mathrm{m}), 7.75(1 \mathrm{H}, \mathrm{s}), 9.59$ $(1 \mathrm{H}, \mathrm{s})$.

Methyl (E)-3-(3-Cyanophenyl)-2-(methoxymethyl)-2-propenoate (6d) To a suspension of $\mathrm{NaH}(680 \mathrm{mg}, 15.6 \mathrm{mmol}$, as a $55 \% \mathrm{w} / \mathrm{w}$ dispersion in mineral oil $)$ in THF $(15 \mathrm{ml})$ was added $\mathrm{MeOH}(0.730 \mathrm{ml}, 18.0 \mathrm{mmol})$ at $0{ }^{\circ} \mathrm{C}$ and the mixture was stirred at room temperature for $30 \mathrm{~min}$. 3-Cyanobenzaldehyde $\mathbf{3}(1.31 \mathrm{~g}, 9.99 \mathrm{mmol})$ and methyl acrylate $\mathbf{5}(1.35 \mathrm{ml}, 15.0 \mathrm{mmol})$ in THF $(15 \mathrm{ml})$ was then added at $0{ }^{\circ} \mathrm{C}$ and the mixture was stirred at room temperature for $2 \mathrm{~h}$. The mixture was concentrated and the resulting residue 
Table 10. ${ }^{1} \mathrm{H}-\mathrm{NMR}$ Data for Cinnamylindoline Derivatives $\mathbf{1 3}$ and $\mathbf{2 2}$

Compd.

${ }^{1} \mathrm{H}-\mathrm{NMR} \delta\left(\right.$ DMSO- $\left.d_{6}\right)$

13a $1.17(3 \mathrm{H}, \mathrm{t}, J=7.4 \mathrm{~Hz}), 1.63-1.83(2 \mathrm{H}, \mathrm{m}), 1.95-2.12(2 \mathrm{H}, \mathrm{m}), 2.30(3 \mathrm{H}, \mathrm{s}), 2.90(1 \mathrm{H}, \mathrm{dd}, J=2.2,16.7 \mathrm{~Hz}), 3.02-3.13(1 \mathrm{H}, \mathrm{m}), 3.19-3.31$ $(1 \mathrm{H}, \mathrm{m}), 3.44-3.88(4 \mathrm{H}, \mathrm{m}), 3.66(1 \mathrm{H}, \mathrm{dd}, J=9.9,16.7 \mathrm{~Hz}), 4.56-4.67(1 \mathrm{H}, \mathrm{m}), 5.11-5.21(1 \mathrm{H}, \mathrm{m}), 6.58(1 \mathrm{H}, \mathrm{dd}, J=6.2,15.8 \mathrm{~Hz}), 6.68$ $(1 \mathrm{H}, \mathrm{d}, J=15.8 \mathrm{~Hz}), 6.87(1 \mathrm{H}, \mathrm{dd}, J=2.1,8.8 \mathrm{~Hz}), 7.00(1 \mathrm{H}, \mathrm{d}, J=2.1 \mathrm{~Hz}), 7.24(1 \mathrm{H}, \mathrm{d}, J=8.8 \mathrm{~Hz}), 7.56(1 \mathrm{H}, \mathrm{t}, J=7.8 \mathrm{~Hz}), 7.70(1 \mathrm{H}, \mathrm{d}, J=7.8 \mathrm{~Hz})$, $7.80(1 \mathrm{H}, \mathrm{d}, J=7.8 \mathrm{~Hz}), 7.93(1 \mathrm{H}, \mathrm{s})$

13b $1.19(3 \mathrm{H}, \mathrm{t}, J=7.4 \mathrm{~Hz}), 1.68-1.78(2 \mathrm{H}, \mathrm{m}), 1.84(3 \mathrm{H}, \mathrm{s}), 1.99-2.08(2 \mathrm{H}, \mathrm{m}), 2.31(3 \mathrm{H}, \mathrm{s}), 2.92(1 \mathrm{H}, \mathrm{dd}, J=3.4,17.0 \mathrm{~Hz}), 3.05-3.29(2 \mathrm{H}, \mathrm{m})$, $3.51-3.83(4 \mathrm{H}, \mathrm{m}), 3.67(1 \mathrm{H}, \mathrm{dd}, J=10.2,17.0 \mathrm{~Hz}), 4.60-4.64(1 \mathrm{H}, \mathrm{m}), 5.06(1 \mathrm{H}, \mathrm{dd}, J=3.4,10.2 \mathrm{~Hz}), 6.55(1 \mathrm{H}, \mathrm{s}), 6.86(1 \mathrm{H}, \mathrm{dd}, J=2.0$, $8.8 \mathrm{~Hz}), 6.98(1 \mathrm{H}, \mathrm{d}, J=2.0 \mathrm{~Hz}), 7.24(1 \mathrm{H}, \mathrm{d}, J=8.8 \mathrm{~Hz}), 7.59-7.70(4 \mathrm{H}, \mathrm{m})$

13c $1.09(3 \mathrm{H}, \mathrm{t}, J=7.5 \mathrm{~Hz}), 1.18(3 \mathrm{H}, \mathrm{t}, J=7.4 \mathrm{~Hz}), 1.68-1.78(2 \mathrm{H}, \mathrm{m}), 2.00-2.08(2 \mathrm{H}, \mathrm{m}), 2.24(1 \mathrm{H}, \mathrm{dt}, J=7.5,14.5 \mathrm{~Hz}), 2.29(3 \mathrm{H}, \mathrm{s}), 2.36(1 \mathrm{H}$, dt, $J=7.5,14.5 \mathrm{~Hz}), 2.89(1 \mathrm{H}, \mathrm{dd}, J=2.2,16.8 \mathrm{~Hz}), 3.04-3.33(2 \mathrm{H}, \mathrm{m}), 3.47-3.58(2 \mathrm{H}, \mathrm{m}), 3.70-3.84(3 \mathrm{H}, \mathrm{m}), 4.58-4.64(1 \mathrm{H}, \mathrm{m}), 5.09$ $(1 \mathrm{H}, \mathrm{dd}, J=2.2,10.3 \mathrm{~Hz}), 6.48(1 \mathrm{H}, \mathrm{s}), 6.87(1 \mathrm{H}, \mathrm{dd}, J=2.2,8.8 \mathrm{~Hz}), 6.98(1 \mathrm{H}, \mathrm{d}, J=2.2 \mathrm{~Hz}), 7.27(1 \mathrm{H}, \mathrm{d}, J=8.8 \mathrm{~Hz}), 7.59-7.68(4 \mathrm{H}, \mathrm{m})$

13d $1.18(3 \mathrm{H}, \mathrm{t}, J=7.3 \mathrm{~Hz}), 1.69-1.88(2 \mathrm{H}, \mathrm{m}), 1.98-2.06(2 \mathrm{H}, \mathrm{m}), 2.29(3 \mathrm{H}, \mathrm{s}), 3.03-3.82(8 \mathrm{H}, \mathrm{m}), 3.20(3 \mathrm{H}, \mathrm{s}), 4.05(1 \mathrm{H}, \mathrm{d}, J=11.5 \mathrm{~Hz})$, 4.09 (1H. d, $J=11.5 \mathrm{~Hz}), 4.58-4.64(1 \mathrm{H}, \mathrm{m}), 5.19(1 \mathrm{H}, \mathrm{dd}, J=2.2,10.2 \mathrm{~Hz}), 6.74(1 \mathrm{H}, \mathrm{s}), 6.86(1 \mathrm{H}, \mathrm{dd}, J=2.4,8.8 \mathrm{~Hz}), 6.98(1 \mathrm{H}, \mathrm{d}, J=2.4 \mathrm{~Hz})$, $7.27(1 \mathrm{H}, \mathrm{d}, J=8.8 \mathrm{~Hz}), 7.60-7.73(4 \mathrm{H}, \mathrm{m})$

13e $\quad 1.19(3 \mathrm{H}, \mathrm{t}, J=7.3 \mathrm{~Hz}), 1.67-1.80(2 \mathrm{H}, \mathrm{m}), 1.99-2.05(2 \mathrm{H}, \mathrm{m}), 2.18(3 \mathrm{H}, \mathrm{s}), 2.29(3 \mathrm{H}, \mathrm{s}), 2.79(1 \mathrm{H}, \mathrm{dd}, J=3.0,16.8 \mathrm{~Hz}), 3.03-3.31(2 \mathrm{H}, \mathrm{m})$, $3.49-3.59(2 \mathrm{H}, \mathrm{m}), 3.65-3.81(3 \mathrm{H}, \mathrm{m}), 4.59-4.63(1 \mathrm{H}, \mathrm{m}), 5.40(1 \mathrm{H}, \mathrm{dt}, J=3.0,9.0 \mathrm{~Hz}), 6.08(1 \mathrm{H}, \mathrm{d}, J=9.0 \mathrm{~Hz}), 6.86(1 \mathrm{H}, \mathrm{d}, J=8.7 \mathrm{~Hz})$, $6.99(1 \mathrm{H}, \mathrm{s}), 7.19(1 \mathrm{H}, \mathrm{d}, J=8.7 \mathrm{~Hz}), 7.57(1 \mathrm{H}, \mathrm{t}, J=7.8 \mathrm{~Hz}), 7.70(1 \mathrm{H}, \mathrm{d}, J=7.8 \mathrm{~Hz}), 7.79(1 \mathrm{H}, \mathrm{d}, J=7.8 \mathrm{~Hz}), 7.84(1 \mathrm{H}, \mathrm{s})$

$13 f \quad 1.11(3 \mathrm{H}, \mathrm{t}, J=7.1 \mathrm{~Hz}), 1.64-1.83(2 \mathrm{H}, \mathrm{m}), 1.97-2.09(2 \mathrm{H}, \mathrm{m}), 2.29(3 \mathrm{H}, \mathrm{s}), 2.90(1 \mathrm{H}, \mathrm{d}, J=16.8 \mathrm{~Hz}), 3.45-3.62(2 \mathrm{H}, \mathrm{m}), 3.65-3.85(2 \mathrm{H}, \mathrm{m})$, $3.67(1 \mathrm{H}, \mathrm{dd}, J=10.3,16.8 \mathrm{~Hz}), 3.88-4.06(2 \mathrm{H}, \mathrm{m}), 4.28(1 \mathrm{H}, \mathrm{d}, J=14.0 \mathrm{~Hz}), 4.43(1 \mathrm{H}, \mathrm{d}, J=14.0 \mathrm{~Hz}), 4.58-4.67(1 \mathrm{H}, \mathrm{m}), 5.20-5.28(1 \mathrm{H}, \mathrm{m})$, $6.58(1 \mathrm{H}, \mathrm{dd}, J=6.6,15.9 \mathrm{~Hz}), 6.67(1 \mathrm{H}, \mathrm{d}, J=15.9 \mathrm{~Hz}), 6.89(1 \mathrm{H}, \mathrm{dd}, J=1.3,8.9 \mathrm{~Hz}), 7.02(1 \mathrm{H}, \mathrm{d}, J=1.3 \mathrm{~Hz}), 7.24(1 \mathrm{H}, \mathrm{d}, J=8.9 \mathrm{~Hz}), 7.56$ $(1 \mathrm{H}, \mathrm{t}, J=7.5 \mathrm{~Hz}), 7.69(1 \mathrm{H}, \mathrm{d}, J=7.5 \mathrm{~Hz}), 7.80(1 \mathrm{H}, \mathrm{d}, J=7.5 \mathrm{~Hz}), 7.91(1 \mathrm{H} . \mathrm{s})$

$13 \mathrm{~g} \quad 1.65-1.82(2 \mathrm{H}, \mathrm{m}), 1.96-2.10(2 \mathrm{H}, \mathrm{m}), 2.16(3 \mathrm{H}, \mathrm{s}), 2.30(3 \mathrm{H}, \mathrm{s}), 2.88(1 \mathrm{H}, \mathrm{d}, J=16.5 \mathrm{~Hz}), 3.46-3.64(3 \mathrm{H}, \mathrm{m}), 3.68-3.87(2 \mathrm{H}, \mathrm{m})$, $4.58-4.66(1 \mathrm{H}, \mathrm{m}), 5.23-5.31(1 \mathrm{H}, \mathrm{m}), 6.57(2 \mathrm{H}, \mathrm{s}), 684(1 \mathrm{H}, \mathrm{d}, J=9.2 \mathrm{~Hz}), 6.96(1 \mathrm{H}, \mathrm{s}), 7.55(1 \mathrm{H}, \mathrm{t}, J=7.8 \mathrm{~Hz}), 7.70(1 \mathrm{H}, \mathrm{d}, J=7.8 \mathrm{~Hz})$, $7.79(1 \mathrm{H}, \mathrm{d}, J=7.8 \mathrm{~Hz}), 7.94(1 \mathrm{H}, \mathrm{s}), 7.99(1 \mathrm{H}, \mathrm{d}, J=9.2 \mathrm{~Hz})$

$13 \mathrm{i} \quad 1.65-1.82(2 \mathrm{H}, \mathrm{m}), 1.96-2.11(2 \mathrm{H}, \mathrm{m}), 2.30(3 \mathrm{H}, \mathrm{s}), 2.88(1 \mathrm{H}, \mathrm{d}, J=16.2 \mathrm{~Hz}), 3.45-3.63(3 \mathrm{H}, \mathrm{m}), 3.67-3.87(2 \mathrm{H}, \mathrm{m}), 3.96-4.11(1 \mathrm{H}, \mathrm{m})$, $4.32-4.44(1 \mathrm{H}, \mathrm{m}), 4.58-4.67(1 \mathrm{H}, \mathrm{m}), 5.18-5.32(1 \mathrm{H}, \mathrm{m}), 6.50-6.62(2 \mathrm{H}, \mathrm{m}), 6.87(1 \mathrm{H}, \mathrm{dd}, J=2.1,8.8 \mathrm{~Hz}), 6.98(1 \mathrm{H}, \mathrm{d}, J=2.1 \mathrm{~Hz})$, $7.56(1 \mathrm{H}, \mathrm{t}, J=7.9 \mathrm{~Hz}), 7.70(1 \mathrm{H}, \mathrm{d}, J=7.9 \mathrm{~Hz}), 7.79(1 \mathrm{H}, \mathrm{d}, J=7.9 \mathrm{~Hz}), 7.92(1 \mathrm{H}, \mathrm{s}), 7.98-8.07(1 \mathrm{H}, \mathrm{m})$

13j $\quad 1.65-1.82(2 \mathrm{H}, \mathrm{m}), 1.97-2.01(2 \mathrm{H}, \mathrm{m}), 2.30(3 \mathrm{H}, \mathrm{s}), 2.88(1 \mathrm{H}, \mathrm{d}, J=16.0 \mathrm{~Hz}), 3.46-3.61(2 \mathrm{H}, \mathrm{m}), 3.65-3.86(2 \mathrm{H}, \mathrm{m}), 3.67(1 \mathrm{H}, \mathrm{dd}, J=10.1$, $16.0 \mathrm{~Hz}), 4.01(1 \mathrm{H}, \mathrm{d}, J=14.2 \mathrm{~Hz}), 4.30(1 \mathrm{H}, \mathrm{d}, J=14.2 \mathrm{~Hz}), 4.58-4.67(1 \mathrm{H}, \mathrm{m}), 5.19-5.27(1 \mathrm{H}, \mathrm{m}), 6.59(1 \mathrm{H}, \mathrm{dd}, J=6.0,15.8 \mathrm{~Hz}), 6.67$ $(1 \mathrm{H}, \mathrm{d}, J=15.8 \mathrm{~Hz}), 6.89(1 \mathrm{H}, \mathrm{dd}, J=2.4,8.8 \mathrm{~Hz}), 7.01(1 \mathrm{H}, \mathrm{d}, J=2.4 \mathrm{~Hz}), 7.24(1 \mathrm{H}, \mathrm{d}, J=8.8 \mathrm{~Hz}), 7.56(1 \mathrm{H}, \mathrm{t}, J=8.0 \mathrm{~Hz}), 7.69(1 \mathrm{H}, \mathrm{d}, J=8.0 \mathrm{~Hz})$, $7.80(1 \mathrm{H}, \mathrm{d}, J=8.0 \mathrm{~Hz}), 7.92(1 \mathrm{H}, \mathrm{s})$

$13 \mathrm{k} \quad 1.11(3 \mathrm{H}, \mathrm{t}, J=7.1 \mathrm{~Hz}), 1.72-1.89(2 \mathrm{H}, \mathrm{m}), 1.96-2.11(2 \mathrm{H}, \mathrm{m}), 2.30(3 \mathrm{H}, \mathrm{s}), 2.92(1 \mathrm{H}, \mathrm{dd}, J=2.1,16.9 \mathrm{~Hz}), 3.51-3.80(4 \mathrm{H}, \mathrm{m}), 3.67$ $(1 \mathrm{H}, \mathrm{dd}, J=9.9,16.9 \mathrm{~Hz}), 3.90-4.07(2 \mathrm{H}, \mathrm{m}), 4.44(1 \mathrm{H}, \mathrm{d}, J=14.3 \mathrm{~Hz}), 4.54(1 \mathrm{H}, \mathrm{d}, J=14.3 \mathrm{~Hz}), 4.68-4.77(1 \mathrm{H}, \mathrm{m}), 5.24-5.33(1 \mathrm{H}, \mathrm{m})$, $6.59(1 \mathrm{H}, \mathrm{dd}, J=6.2,15.8 \mathrm{~Hz}), 6.68(1 \mathrm{H}, \mathrm{d}, J=15.8 \mathrm{~Hz}), 7.33(1 \mathrm{H}, \mathrm{s}), 7.34(1 \mathrm{H}, \mathrm{s}), 7.57(1 \mathrm{H}, \mathrm{t}, J=7.9 \mathrm{~Hz}), 7.70(1 \mathrm{H}, \mathrm{d}, J=7.9 \mathrm{~Hz}), 7.81(1 \mathrm{H}, \mathrm{d}$, $J=7.9 \mathrm{~Hz}), 7.92(1 \mathrm{H}, \mathrm{s})$

$1311.10(3 \mathrm{H}, \mathrm{t}, J=7.1 \mathrm{~Hz}), 1.71-1.90(2 \mathrm{H}, \mathrm{m}), 1.96-2.14(2 \mathrm{H}, \mathrm{m}), 2.30(3 \mathrm{H}, \mathrm{s}), 2.95(1 \mathrm{H}, \mathrm{dd}, J=2.6,16.8 \mathrm{~Hz}), 3.51-3.82(4 \mathrm{H}, \mathrm{m}), 3.69$ $(1 \mathrm{H}, \mathrm{dd}, J=10.0,16.8 \mathrm{~Hz}), 3.85-3.95(1 \mathrm{H}, \mathrm{m}), 3.98-4.08(1 \mathrm{H}, \mathrm{m}), 4.41(1 \mathrm{H}, \mathrm{d}, J=14.3 \mathrm{~Hz}), 4.50(1 \mathrm{H}, \mathrm{d}, J=14.3 \mathrm{~Hz}), 4.69-4.77(1 \mathrm{H}, \mathrm{m})$, $5.27-5.35(1 \mathrm{H}, \mathrm{m}), 6.62(1 \mathrm{H}, \mathrm{dd}, J=5.9,15.8 \mathrm{~Hz}), 6.69(1 \mathrm{H}, \mathrm{d}, J=15.8 \mathrm{~Hz}), 7.19(1 \mathrm{H}, \mathrm{d}, J=8.9 \mathrm{~Hz}), 7.23(1 \mathrm{H}, \mathrm{d}, J=8.9 \mathrm{~Hz}), 7.57(1 \mathrm{H}, \mathrm{t}$, $J=8.0 \mathrm{~Hz}), 7.71(1 \mathrm{H}, \mathrm{d}, J=8.0 \mathrm{~Hz}), 7.81(1 \mathrm{H}, \mathrm{d}, J=8.0 \mathrm{~Hz}), 7.95(1 \mathrm{H}, \mathrm{s})$

$13 \mathrm{~m} 1.11(3 \mathrm{H}, \mathrm{t}, J=7.3 \mathrm{~Hz}), 1.66-1.85(2 \mathrm{H}, \mathrm{m}), 1.98-2.01(2 \mathrm{H}, \mathrm{m}), 2.30(3 \mathrm{H}, \mathrm{s}), 3.00(1 \mathrm{H}, \mathrm{d}, J=16.5 \mathrm{~Hz}), 3.47-3.61(2 \mathrm{H}, \mathrm{m}), 3.63-3.85(3 \mathrm{H}, \mathrm{m})$, $3.89-4.08(2 \mathrm{H}, \mathrm{m}), 4.40(1 \mathrm{H}, \mathrm{d}, J=14.3 \mathrm{~Hz}), 4.50(1 \mathrm{H}, \mathrm{d}, J=14.3 \mathrm{~Hz}), 4.57-4.65(1 \mathrm{H}, \mathrm{m}), 5.29-5.36(1 \mathrm{H}, \mathrm{m}), 6.60(1 \mathrm{H}, \mathrm{dd}, J=6.3,15.8 \mathrm{~Hz})$, $6.70(1 \mathrm{H}, \mathrm{d}, J=15.8 \mathrm{~Hz}), 7.07(1 \mathrm{H}, \mathrm{d}, J=8.7 \mathrm{~Hz}), 7.19(1 \mathrm{H}, \mathrm{t}, J=8.7 \mathrm{~Hz}), 7.57(1 \mathrm{H}, \mathrm{t}, J=7.9 \mathrm{~Hz}), 7.70(1 \mathrm{H}, \mathrm{d}, J=7.9 \mathrm{~Hz}), 7.81(1 \mathrm{H}, \mathrm{d}, J=7.9 \mathrm{~Hz})$, $7.93(1 \mathrm{H}, \mathrm{s})$

13n $1.12(3 \mathrm{H}, \mathrm{t}, J=7.1 \mathrm{~Hz}), 1.69-1.85(2 \mathrm{H}, \mathrm{m}), 1.97-2.10(2 \mathrm{H}, \mathrm{m}), 2.30(3 \mathrm{H}, \mathrm{s}), 2.90(1 \mathrm{H}, \mathrm{d}, J=15.3 \mathrm{~Hz}), 3.46-3.86(5 \mathrm{H}, \mathrm{m}), 3.90-4.08(2 \mathrm{H}, \mathrm{m})$, $4.43(1 \mathrm{H}, \mathrm{d}, J=14.4 \mathrm{~Hz}), 4.54(1 \mathrm{H}, \mathrm{d}, J=14.4 \mathrm{~Hz}), 4.57-4.65(1 \mathrm{H}, \mathrm{m}), 5.24-5.32(1 \mathrm{H}, \mathrm{m}), 6.60(1 \mathrm{H}, \mathrm{dd}, J=6.2,15.8 \mathrm{~Hz}), 6.68(1 \mathrm{H}, \mathrm{d}$, $J=15.8 \mathrm{~Hz}), 7.14(1 \mathrm{H}, \mathrm{d}, J=11.6 \mathrm{~Hz}), 7.32(1 \mathrm{H}, \mathrm{d}, J=8.3 \mathrm{~Hz}), 7.57(1 \mathrm{H}, \mathrm{t}, J=7.8 \mathrm{~Hz}), 7.71(1 \mathrm{H}, \mathrm{d}, J=7.8 \mathrm{~Hz}), 7.80(1 \mathrm{H} . \mathrm{d}, J=7.8 \mathrm{~Hz}), 7.95$ $(1 \mathrm{H}, \mathrm{s})$

$13 \mathrm{o} \quad 1.10(3 \mathrm{H}, \mathrm{t}, J=7.1 \mathrm{~Hz}), 1.74-1.86(2 \mathrm{H}, \mathrm{m}), 2.02-2.11(2 \mathrm{H}, \mathrm{m}), 2.31(3 \mathrm{H}, \mathrm{s}), 3.00(1 \mathrm{H}, \mathrm{d}, J=16.9 \mathrm{~Hz}), 3.56-3.70(4 \mathrm{H}, \mathrm{m}), 3.77(1 \mathrm{H}, \mathrm{dd}$, $J=9.4,16.9 \mathrm{~Hz}), 3.89-4.03(2 \mathrm{H}, \mathrm{m}), 4.49(1 \mathrm{H}, \mathrm{d}, J=13.0 \mathrm{~Hz}), 4.57(1 \mathrm{H}, \mathrm{d}, J=13.0 \mathrm{~Hz}), 4.88-4.90(1 \mathrm{H}, \mathrm{m}), 5.33(1 \mathrm{H}, \mathrm{t}, J=7.1 \mathrm{~Hz}), 6.61(1 \mathrm{H}$, dd, $J=6.1,15.8 \mathrm{~Hz}), 6.69(1 \mathrm{H}, \mathrm{d}, J=15.8 \mathrm{~Hz}), 7.47(1 \mathrm{H}, \mathrm{s}), 7.50(1 \mathrm{H}, \mathrm{s}), 7.57(1 \mathrm{H}, \mathrm{t}, J=7.8 \mathrm{~Hz}), 7.72(1 \mathrm{H}, \mathrm{d}, J=7.8 \mathrm{~Hz}), 7.81(1 \mathrm{H}, \mathrm{d}, J=7.8 \mathrm{~Hz})$, $7.96(1 \mathrm{H}, \mathrm{s})$

13p $1.09(3 \mathrm{H}, \mathrm{t}, J=7.1 \mathrm{~Hz}), 1.75-1.85(2 \mathrm{H}, \mathrm{m}), 2.01-2.12(2 \mathrm{H}, \mathrm{m}), 2.30(3 \mathrm{H}, \mathrm{s}), 3.10(1 \mathrm{H}, \mathrm{d}, J=18.1 \mathrm{~Hz}), 3.56-3.71(4 \mathrm{H}, \mathrm{m}), 3.80-4.06(3 \mathrm{H}, \mathrm{m})$, $4.44(1 \mathrm{H}, \mathrm{d}, J=14.4 \mathrm{~Hz}), 4.53(1 \mathrm{H}, \mathrm{d}, J=14.4 \mathrm{~Hz}), 4.86-4.88(1 \mathrm{H}, \mathrm{m}), 5.26-5.34(1 \mathrm{H}, \mathrm{m}), 6.62(1 \mathrm{H}, \mathrm{dd}, J=6.1,15.8 \mathrm{~Hz}), 6.68(1 \mathrm{H}, \mathrm{d}$, $J=15.8 \mathrm{~Hz}), 7.29(1 \mathrm{H}, \mathrm{d}, J=9.2 \mathrm{~Hz}), 7.54(1 \mathrm{H}, \mathrm{d}, J=9.2 \mathrm{~Hz}), 7.57(1 \mathrm{H}, \mathrm{t}, J=7.8 \mathrm{~Hz}), 7.69(1 \mathrm{H}, \mathrm{d}, J=7.8 \mathrm{~Hz}), 7.81(1 \mathrm{H}, \mathrm{d}, J=7.8 \mathrm{~Hz}), 7.92(1 \mathrm{H}, \mathrm{s})$

$13 q \quad 1.12(3 \mathrm{H}, \mathrm{t}, J=7.1 \mathrm{~Hz}), 1.70-1.85(2 \mathrm{H}, \mathrm{m}), 1.96-2.10(2 \mathrm{H}, \mathrm{m}), 2.17(3 \mathrm{H}, \mathrm{s}), 2.31(3 \mathrm{H}, \mathrm{s}), 2.87(1 \mathrm{H}, \mathrm{d}, J=15.6 \mathrm{~Hz}), 3.52-3.84(5 \mathrm{H}, \mathrm{m})$, $3.90-4.08(2 \mathrm{H}, \mathrm{m}), 4.24(1 \mathrm{H}, \mathrm{d}, J=14.2 \mathrm{~Hz}), 4.43(1 \mathrm{H}, \mathrm{d}, J=14.2 \mathrm{~Hz}), 4.60-4.69(1 \mathrm{H}, \mathrm{m}), 5.18-5.26(1 \mathrm{H}, \mathrm{m}), 6.55(1 \mathrm{H}, \mathrm{dd}, J=6.2,15.8 \mathrm{~Hz})$, $6.66(1 \mathrm{H}, \mathrm{d}, J=15.8 \mathrm{~Hz}), 7.06(1 \mathrm{H}, \mathrm{s}), 7.16(1 \mathrm{H}, \mathrm{s}), 7.60(1 \mathrm{H}, \mathrm{t}, J=7.8 \mathrm{~Hz}), 7.68(1 \mathrm{H}, \mathrm{d}, J=7.8 \mathrm{~Hz}), 7.80(1 \mathrm{H} . \mathrm{d}, J=7.8 \mathrm{~Hz}), 7.87(1 \mathrm{H}, \mathrm{s})$

$13 \mathrm{r} \quad 1.11(3 \mathrm{H}, \mathrm{t}, J=7.1 \mathrm{~Hz}), 1.68-1.85(2 \mathrm{H}, \mathrm{m}), 1.93-2.13(2 \mathrm{H}, \mathrm{m}), 2.10(3 \mathrm{H}, \mathrm{s}), 2.32(3 \mathrm{H}, \mathrm{s}), 2.89(1 \mathrm{H}, \mathrm{d}, J=16.0 \mathrm{~Hz}), 3.46-3.84(5 \mathrm{H}, \mathrm{m}), 3.88-$ $4.09(2 \mathrm{H}, \mathrm{m}), 4.24(1 \mathrm{H}, \mathrm{d}, J=14.7 \mathrm{~Hz}), 4.42(1 \mathrm{H}, \mathrm{d}, J=14.7 \mathrm{~Hz}), 4.58-4.69(1 \mathrm{H}, \mathrm{m}), 5.20-5.30(1 \mathrm{H}, \mathrm{m}), 6.60(1 \mathrm{H}, \mathrm{dd}, J=6.1,15.8 \mathrm{~Hz}), 6.68$ $(1 \mathrm{H}, \mathrm{d}, J=15.8 \mathrm{~Hz}), 6.95(1 \mathrm{H}, \mathrm{d}, J=8.8 \mathrm{~Hz}), 7.11(1 \mathrm{H}, \mathrm{d}, J=8.8 \mathrm{~Hz}), 7.56(1 \mathrm{H}, \mathrm{t}, J=7.8 \mathrm{~Hz}), 7.73(1 \mathrm{H}, \mathrm{d}, J=7.8 \mathrm{~Hz}), 7.80(1 \mathrm{H}, \mathrm{d}, J=7.8 \mathrm{~Hz}), 7.96$ $(1 \mathrm{H}, \mathrm{s})$

$13 \mathrm{t} \quad 1.11(3 \mathrm{H}, \mathrm{t}, J=7.2 \mathrm{~Hz}), 1.28(3 \mathrm{H}, \mathrm{t}, J=7.1 \mathrm{~Hz}), 1.81-1.86(2 \mathrm{H}, \mathrm{m}), 1.96-2.02(2 \mathrm{H}, \mathrm{m}), 2.30(3 \mathrm{H}, \mathrm{s}), 2.96(1 \mathrm{H}, \mathrm{dd}, J=1.8,14.3 \mathrm{~Hz}), 3.62-3.77$ $(5 \mathrm{H}, \mathrm{m}), 3.96-4.00(2 \mathrm{H}, \mathrm{m}), 4.27(2 \mathrm{H}, \mathrm{q}, J=7.2 \mathrm{~Hz}), 4.41(1 \mathrm{H} . \mathrm{d}, J=14.3 \mathrm{~Hz}), 4.51(1 \mathrm{H}, \mathrm{d}, J=14.3 \mathrm{~Hz}), 4.80-4.81(1 \mathrm{H}, \mathrm{m}), 5.29-5.30(1 \mathrm{H}$, $\mathrm{m}), 6.58(1 \mathrm{H}, \mathrm{dd}, J=6.0,15.8 \mathrm{~Hz}), 6.68(1 \mathrm{H}, \mathrm{d}, J=15.8 \mathrm{~Hz}), 7.32(1 \mathrm{H}, \mathrm{s}), 7.57(1 \mathrm{H}, \mathrm{t}, J=7.7 \mathrm{~Hz}), 7.61(1 \mathrm{H}, \mathrm{s}), 7.69(1 \mathrm{H}, \mathrm{d}, J=7.7 \mathrm{~Hz}), 7.81(1 \mathrm{H}$, d, $J=7.7 \mathrm{~Hz}), 7.91(1 \mathrm{H}, \mathrm{s})$

13u $1.12(3 \mathrm{H}, \mathrm{t}, J=7.1 \mathrm{~Hz}), 1.78-1.92(2 \mathrm{H}, \mathrm{m}), 2.00-2.10(2 \mathrm{H}, \mathrm{m}), 2.30(3 \mathrm{H}, \mathrm{s}), 2.95(1 \mathrm{H}, \mathrm{d}, J=17.0 \mathrm{~Hz}), 3.47-3.59(2 \mathrm{H}, \mathrm{m}), 3.69-3.75(2 \mathrm{H}, \mathrm{m})$, $3.82-3.87(1 \mathrm{H}, \mathrm{m}), 3.96-4.05(2 \mathrm{H}, \mathrm{m}), 4.34(1 \mathrm{H}, \mathrm{d}, J=15.0 \mathrm{~Hz}), 4.48(1 \mathrm{H}, \mathrm{d}, J=15.0 \mathrm{~Hz}), 4.78-4.81(1 \mathrm{H}, \mathrm{m}), 5.26(1 \mathrm{H}, \mathrm{t}, J=6.9 \mathrm{~Hz}), 6.59$ $(1 \mathrm{H}, \mathrm{dd}, J=6.0,15.8 \mathrm{~Hz}), 6.68(1 \mathrm{H}, \mathrm{d}, J=15.8 \mathrm{~Hz}), 7.29(1 \mathrm{H}, \mathrm{s}), 7.57(1 \mathrm{H}, \mathrm{t}, J=7.9 \mathrm{~Hz}), 7.69(1 \mathrm{H}, \mathrm{d}, J=7.9 \mathrm{~Hz}), 7.71(1 \mathrm{H}, \mathrm{s}), 7.81(1 \mathrm{H}, \mathrm{d}$, $J=7.9 \mathrm{~Hz}), 7.92(1 \mathrm{H}, \mathrm{s})$ 
Table 10. (Continued)

Compd

${ }^{1} \mathrm{H}-\mathrm{NMR} \delta\left(\mathrm{DMSO}-d_{6}\right)$

22a $1.17(3 \mathrm{H}, \mathrm{t}, J=7.4 \mathrm{~Hz}), 1.65-1.83(2 \mathrm{H}, \mathrm{m}), 1.96-2.11(2 \mathrm{H}, \mathrm{m}), 2.30(3 \mathrm{H}, \mathrm{s}), 2.86(1 \mathrm{H}, \mathrm{dd}, J=2.4,17.0 \mathrm{~Hz}), 3.00-3.12(1 \mathrm{H}, \mathrm{m}), 3.19-3.30$ $(1 \mathrm{H}, \mathrm{m}), 3.46-3.61(2 \mathrm{H}, \mathrm{m}), 3.65(1 \mathrm{H}, \mathrm{dd}, J=9.9,17.0 \mathrm{~Hz}), 3.67-3.86(2 \mathrm{H}, \mathrm{m}), 4.57-4.66(1 \mathrm{H}, \mathrm{m}), 5.09-5.18(1 \mathrm{H}, \mathrm{m}), 6.52(1 \mathrm{H}, \mathrm{dd}, J=6.9$, $16.0 \mathrm{~Hz}), 682(1 \mathrm{H}, \mathrm{d}, J=16.0 \mathrm{~Hz}), 6.86(1 \mathrm{H}, \mathrm{dd}, J=2.4,8.8 \mathrm{~Hz}), 7.01(1 \mathrm{H}, \mathrm{d}, J=2.4 \mathrm{~Hz}), 7.06(1 \mathrm{H}, \mathrm{d}, J=8.6 \mathrm{~Hz}), 7.23(1 \mathrm{H}, \mathrm{d}, J=8.8 \mathrm{~Hz}), 7.62$ $(1 \mathrm{H}, \mathrm{dd}, J=2.4,8.6 \mathrm{~Hz}), 7.97(1 \mathrm{H}, \mathrm{d}, J=2.4 \mathrm{~Hz})$

22b $\quad 1.17(3 \mathrm{H}, \mathrm{t}, J=7.4 \mathrm{~Hz}), 1.64-1.82(2 \mathrm{H}, \mathrm{m}), 1.95-2.11(2 \mathrm{H}, \mathrm{m}), 2.30(3 \mathrm{H}, \mathrm{s}), 2.87(1 \mathrm{H}, \mathrm{dd}, J=2.4,16.8 \mathrm{~Hz}), 3.00-3.12(1 \mathrm{H}, \mathrm{m}), 3.18-3.30$ $(1 \mathrm{H}, \mathrm{m}), 3.44-3.87(4 \mathrm{H}, \mathrm{m}), 3.66(1 \mathrm{H}, \mathrm{dd}, J=10.0,16.8 \mathrm{~Hz}), 3.92(3 \mathrm{H}, \mathrm{s}), 4.56-4.67(1 \mathrm{H}, \mathrm{m}), 5.11-5.20(1 \mathrm{H}, \mathrm{m}), 6.56(1 \mathrm{H}, \mathrm{dd}, J=7.0$, $16.1 \mathrm{~Hz}), 6.85(1 \mathrm{H}, \mathrm{d}, J=16.1 \mathrm{~Hz}), 6.86(1 \mathrm{H}, \mathrm{dd}, J=2.3,8.9 \mathrm{~Hz}), 7.00(1 \mathrm{H}, \mathrm{d}, J=2.3 \mathrm{~Hz}), 7.23(1 \mathrm{H}, \mathrm{d}, J=8.8 \mathrm{~Hz}), 7.24(1 \mathrm{H}, \mathrm{d}, J=8.9 \mathrm{~Hz}), 7.82$ $(1 \mathrm{H}, \mathrm{dd}, J=2.4,8.8 \mathrm{~Hz}), 8.05(1 \mathrm{H}, \mathrm{d}, J=2.4 \mathrm{~Hz})$

$22 \mathrm{c} 1.10(3 \mathrm{H}, \mathrm{t}, J=7.1 \mathrm{~Hz}), 1.65-1.82(2 \mathrm{H}, \mathrm{m}), 1.96-2.10(2 \mathrm{H}, \mathrm{m}), 2.30(3 \mathrm{H}, \mathrm{s}), 2.87(1 \mathrm{H}, \mathrm{d}, J=16.4 \mathrm{~Hz}), 3.46-3.62(2 \mathrm{H}, \mathrm{m}), 3.67(1 \mathrm{H}, \mathrm{dd}$, $J=9.6,16.4 \mathrm{~Hz}), 3.68-3.85(2 \mathrm{H}, \mathrm{m}), 3.87-4.06(2 \mathrm{H}, \mathrm{m}), 4.26(1 \mathrm{H}, \mathrm{d}, J=14.1 \mathrm{~Hz}), 4.42(1 \mathrm{H}, \mathrm{d}, J=14.1 \mathrm{~Hz}), 4.60-4.67(1 \mathrm{H}, \mathrm{m}), 5.18-5.27$ $(1 \mathrm{H}, \mathrm{m}), 6.53(1 \mathrm{H}, \mathrm{dd}, J=6.9,15.8 \mathrm{~Hz}), 6.82(1 \mathrm{H}, \mathrm{d}, J=15.8 \mathrm{~Hz}), 6.89(1 \mathrm{H}, \mathrm{dd}, J=2.3,8.8 \mathrm{~Hz}), 7.02(1 \mathrm{H}, \mathrm{s}), 7.08(1 \mathrm{H}, \mathrm{d}, J=8.6 \mathrm{~Hz}), 7.23$ $(1 \mathrm{H}, \mathrm{d}, J=8.8 \mathrm{~Hz}), 7.64(1 \mathrm{H}, \mathrm{d}, J=8.6 \mathrm{~Hz}), 7.98(1 \mathrm{H}, \mathrm{s})$

$22 f \quad 1.65-1.82(2 \mathrm{H}, \mathrm{m}), 1.96-2.10(2 \mathrm{H}, \mathrm{m}), 2.30(3 \mathrm{H}, \mathrm{s}), 2.85(1 \mathrm{H}, \mathrm{d}, J=15.8 \mathrm{~Hz}), 3.44-3.61(2 \mathrm{H}, \mathrm{m}), 3.66(1 \mathrm{H}, \mathrm{dd}, J=10.1,15.8 \mathrm{~Hz}), 3.68-3.87$ $(2 \mathrm{H}, \mathrm{m}), 4.02(1 \mathrm{H}, \mathrm{d}, J=14.2 \mathrm{~Hz}), 4.31(1 \mathrm{H}, \mathrm{d}, J=14.2 \mathrm{~Hz}), 4.58-4.67(1 \mathrm{H}, \mathrm{m}), 5.15-5.25(1 \mathrm{H}, \mathrm{m}), 6.53(1 \mathrm{H}, \mathrm{dd}, J=6.8,15.9 \mathrm{~Hz}), 6.82$ $(1 \mathrm{H}, \mathrm{d}, J=15.9 \mathrm{~Hz}), 6.88(1 \mathrm{H}, \mathrm{dd}, J=2.4,8.8 \mathrm{~Hz}), 7.01(1 \mathrm{H}, \mathrm{d}, J=2.4 \mathrm{~Hz}), 7.08(1 \mathrm{H}, \mathrm{d}, J=8.6 \mathrm{~Hz}), 7.24(1 \mathrm{H}, \mathrm{d}, J=8.8 \mathrm{~Hz}), 7.63(1 \mathrm{H}, \mathrm{dd}, J=2.2$, $8.6 \mathrm{~Hz}), 7.98(1 \mathrm{H}, \mathrm{d}, J=2.2 \mathrm{~Hz})$

$22 \mathrm{~g} \quad 1.72-1.90(2 \mathrm{H}, \mathrm{m}), 1.96-2.13(2 \mathrm{H}, \mathrm{m}), 2.30(3 \mathrm{H}, \mathrm{s}), 2.87(1 \mathrm{H}, \mathrm{d}, J=16.4 \mathrm{~Hz}), 3.51-3.80(4 \mathrm{H}, \mathrm{m}), 3.68(1 \mathrm{H}, \mathrm{dd}, J=9.6,16.4 \mathrm{~Hz}), 4.10$ $(1 \mathrm{H}, \mathrm{d}, J=14.0 \mathrm{~Hz}), 4.32(1 \mathrm{H}, \mathrm{d}, J=14.0 \mathrm{~Hz}), 4.67-4.77(1 \mathrm{H}, \mathrm{m}), 5.25-5.33(1 \mathrm{H}, \mathrm{m}), 6.54(1 \mathrm{H}, \mathrm{dd}, J=6.9,15.9 \mathrm{~Hz}), 6.84(1 \mathrm{H}, \mathrm{d}, J=15.9 \mathrm{~Hz})$, $7.06(1 \mathrm{H}, \mathrm{d}, J=8.7 \mathrm{~Hz}), 7.17(1 \mathrm{H}, \mathrm{d}, J=8.8 \mathrm{~Hz}), 7.23(1 \mathrm{H}, \mathrm{d}, J=8.8 \mathrm{~Hz}), 7.62(1 \mathrm{H}, \mathrm{dd}, J=2.2,8.7 \mathrm{~Hz}), 7.99(1 \mathrm{H}, \mathrm{d}, J=2.2 \mathrm{~Hz})$

$22 \mathrm{~h} \quad 1.73-1.88(2 \mathrm{H}, \mathrm{m}), 1.97-2.11(2 \mathrm{H}, \mathrm{m}), 2.30(3 \mathrm{H}, \mathrm{s}), 2.87(1 \mathrm{H}, \mathrm{dd}, J=1.2,16.7 \mathrm{~Hz}), 3.49-3.80(5 \mathrm{H}, \mathrm{m}), 4.14(1 \mathrm{H}, \mathrm{d}, J=14.2 \mathrm{~Hz}), 4.38$ $(1 \mathrm{H}, \mathrm{d}, J=14.2 \mathrm{~Hz}), 4.68-4.76(1 \mathrm{H}, \mathrm{m}), 5.22-5.28(1 \mathrm{H}, \mathrm{m}), 6.53(1 \mathrm{H}, \mathrm{dd}, J=6.9,15.7 \mathrm{~Hz}), 6.82(1 \mathrm{H}, \mathrm{d}, J=15.7 \mathrm{~Hz}), 7.05(1 \mathrm{H}, \mathrm{d}, J=8.7 \mathrm{~Hz})$, $7.32(2 \mathrm{H}, \mathrm{s}), 7.62(1 \mathrm{H}, \mathrm{dd}, J=2.3,8.7 \mathrm{~Hz}), 7.97(1 \mathrm{H}, \mathrm{d}, J=2.3 \mathrm{~Hz})$

was diluted with EtOAc. The organic layer was washed with $\mathrm{H}_{2} \mathrm{O}$ and brine The organic layer was dried and concentrated. The resulting residue was chromatographed on a silica gel column (toluene/EtOAc $=20 / 1$ ) to give $\mathbf{6 d}$ $(0.720 \mathrm{~g}, 3.11 \mathrm{mmol}, 31 \%)$ as a colorless solid. ${ }^{1} \mathrm{H}-\mathrm{NMR}\left(\mathrm{CDCl}_{3}\right) \delta: 3.46$ $(3 \mathrm{H}, \mathrm{s}), 3.88(3 \mathrm{H}, \mathrm{s}), 4.19(2 \mathrm{H}, \mathrm{s}), 7.55(1 \mathrm{H}, \mathrm{t}, J=7.8 \mathrm{~Hz}), 7.68(1 \mathrm{H}, \mathrm{d}$, $J=7.8 \mathrm{~Hz}), 7.75(1 \mathrm{H}, \mathrm{d}, J=7.8 \mathrm{~Hz}), 7.84(1 \mathrm{H}, \mathrm{s}), 7.87(1 \mathrm{H}, \mathrm{s})$.

Ethyl (E)-3-(3-Cyanophenyl)-3-methylacrylate (6e) A solution of 3cyanoacetophenone $7(2.90 \mathrm{~g}, 20.0 \mathrm{mmol})$ and (carbethoxymethylene)triphenylphosphorane $(17.4 \mathrm{~g}, 49.9 \mathrm{mmol})$ in xylene $(150 \mathrm{ml})$ was refluxed for $4 \mathrm{~h}$ with stirring and the mixture was concentrated. The resulting residue was chromatographed on a silica gel column (hexane/EtOAc $=4 / 1$ ) to give $6 \mathrm{e}$ $(1.61 \mathrm{~g}, 7.48 \mathrm{mmol}, 37 \%)$ as a yellow solid. ${ }^{1} \mathrm{H}-\mathrm{NMR}\left(\mathrm{CDCl}_{3}\right) \delta: 1.33(3 \mathrm{H}, \mathrm{t}$, $J=7.1 \mathrm{~Hz}), 2.56(3 \mathrm{H}, \mathrm{s}), 4.23(2 \mathrm{H}, \mathrm{q}, J=7.1 \mathrm{~Hz}), 6.13(1 \mathrm{H}, \mathrm{s}), 7.50(1 \mathrm{H}, \mathrm{t}$, $J=8.0 \mathrm{~Hz}), 7.64(1 \mathrm{H}, \mathrm{d}, J=8.0 \mathrm{~Hz}), 7.70(1 \mathrm{H}, \mathrm{d}, J=8.0 \mathrm{~Hz}), 7.75(1 \mathrm{H}, \mathrm{s})$

(E)-3-(3-Cyanophenyl)-2-(methoxymethyl)-2-propenal (4d) To a solution of methyl $(E)$-3-(3-cyanophenyl)-2-(methoxymethyl)-2-propenoate $\mathbf{6 d}$ $(2.01 \mathrm{~g}, 8.69 \mathrm{mmol})$ in $\mathrm{CH}_{2} \mathrm{Cl}_{2}(20 \mathrm{ml})$ and hexane $(50 \mathrm{ml})$ was added diisobutylaluminum hydride (DIBAL) $(1.5 \mathrm{M}$ in toluene, $6.70 \mathrm{ml}, 10.1 \mathrm{mmol})$ and $i-\mathrm{Bu}_{3} \mathrm{Al}(1.0 \mathrm{M}$ in hexane, $10.0 \mathrm{ml}, 10.0 \mathrm{mmol})$ at $-78^{\circ} \mathrm{C}$ and the mixture was stirred for $30 \mathrm{~min}$. After adding DIBAL $(1.5 \mathrm{~m}$ in toluene, $6.70 \mathrm{ml}$, $10.1 \mathrm{mmol})$ and $i-\mathrm{Bu}_{3} \mathrm{Al}(1.0 \mathrm{M}$ in hexane, $10.0 \mathrm{ml}, 10.0 \mathrm{mmol})$ at $-78^{\circ} \mathrm{C}$ and the mixture was stirred for $30 \mathrm{~min}$. $\mathrm{MeOH}$ was added, and the mixture was diluted with EtOAc and washed with $1 \mathrm{~N} \mathrm{HCl}, \mathrm{H}_{2} \mathrm{O}, \mathrm{NaHCO}_{3}$ solution, and brine. The organic layer was dried and concentrated. The resulting residue was chromatographed on a silica gel column (hexane/EtOAc $=1 / 1)$ to give a mixture of $(E)$ - and (Z)-3-(3-formylphenyl)-2-(methoxymethyl)-2propen-1-ol. The mixture was dissolved in 1-methyl-2-pyrrolidinone $(30 \mathrm{ml})$ and the solution was added hydroxylamine hydrochloride $(700 \mathrm{mg}$, $10.1 \mathrm{mmol}$ ). The mixture was stirred at $120^{\circ} \mathrm{C}$ for $4 \mathrm{~h}$. After cooling, the mixture was diluted with EtOAc and washed with $\mathrm{H}_{2} \mathrm{O}$, brine. The organic layer was dried and concentrated. The resulting residue was chromatographed on a silica gel column (hexane/EtOAc $=1 / 1)$ to give $(Z)-3-(3-$ cyanophenyl)-2-(methoxymethyl)-2-propen-1-ol $(850 \mathrm{mg}, 4.18 \mathrm{mmol})$ as a yellow solid. To a solution of (Z)-3-(3-cyanophenyl)-2-(methoxymethyl)-2propen-1-ol $(850 \mathrm{mg}, 4.18 \mathrm{mmol})$ in $\mathrm{CH}_{2} \mathrm{Cl}_{2}(10 \mathrm{ml})$ and hexane $(10 \mathrm{ml})$ was added $\mathrm{MnO}_{2}(5.00 \mathrm{~g}, 63.3 \mathrm{mmol})$ and the mixture was stirred at room temperature for $4 \mathrm{~h}$. After adding $\mathrm{MnO}_{2}(5.00 \mathrm{~g}, 63.3 \mathrm{mmol})$ and the mixture was stirred at room temperature for $1 \mathrm{~h} . \mathrm{MnO}_{2}$ was filtered away, and the filtrate was concentrated. The resulting residue was chromatographed on a silica gel column (hexane/EtOAc $=3 / 2)$ to give $\mathbf{4 d}(0.71 \mathrm{~g}, 3.53 \mathrm{mmol}, 41 \%)$ as a pale yellow solid. ${ }^{1} \mathrm{H}-\mathrm{NMR}\left(\mathrm{CDCl}_{3}\right) \delta: 3.45(3 \mathrm{H}, \mathrm{s}), 4.22(2 \mathrm{H}, \mathrm{s}), 7.47$ $(1 \mathrm{H}, \mathrm{s}), 7.60(1 \mathrm{H}, \mathrm{t}, J=7.8 \mathrm{~Hz}), 7.74(1 \mathrm{H}, \mathrm{d}, J=7.8 \mathrm{~Hz}), 7.90(1 \mathrm{H}, \mathrm{d}$, $J=7.8 \mathrm{~Hz}), 7.99(1 \mathrm{H}, \mathrm{s}), 9.67(1 \mathrm{H}, \mathrm{s})$

Another derivative (4e) was similarly prepared.

4e: ${ }^{1} \mathrm{H}-\mathrm{NMR}\left(\mathrm{CDCl}_{3}\right) \delta: 2.63(3 \mathrm{H}, \mathrm{s}), 6.41(1 \mathrm{H}, \mathrm{d}, J=7.5 \mathrm{~Hz}), 7.60(1 \mathrm{H}, \mathrm{t}$,
$J=7.8 \mathrm{~Hz}), 7.76(1 \mathrm{H}, \mathrm{d}, J=7.8 \mathrm{~Hz}), 7.81(1 \mathrm{H}, \mathrm{d}, J=7.8 \mathrm{~Hz}), 7.86(1 \mathrm{H}, \mathrm{s})$, $10.24(1 \mathrm{H}, \mathrm{d}, J=7.5 \mathrm{~Hz})$

$t$-Butyl (E)-4-\{3-[4-(3-Cyanophenyl)-2-hydroxy-3-buten-1-yl]-4-nitrophenoxy $\}$ piperidine-1-carboxylate (9a) To a solution of (E)-3-cyanocinnamaldehyde $4 \mathrm{a}(1.00 \mathrm{~g}, 6.36 \mathrm{mmol})$ and $t$-butyl 4-[4-nitro-3-(trimethylsilylmethyl)phenoxy]piperidine-1-carboxlate $8 \mathbf{a}(2.86 \mathrm{~g}, 7.00 \mathrm{mmol})$ in THF $(50 \mathrm{ml})$ was added a solution of TBAF monohydrate $(0.180 \mathrm{~g}, 0.644 \mathrm{mmol})$ in THF $(10 \mathrm{ml})$ at $-10^{\circ} \mathrm{C}$ and the mixture was stirred at $-10^{\circ} \mathrm{C}$ for $1 \mathrm{~h}$ TBAF $\left(75 \%\right.$ in $\left.\mathrm{H}_{2} \mathrm{O}, 1.19 \mathrm{~g}, 6.07 \mathrm{mmol}\right)$ in THF $(10 \mathrm{ml})$ was then added and the mixture was stirred at $-10^{\circ} \mathrm{C}$ for $1 \mathrm{~h} . \mathrm{NH}_{4} \mathrm{Cl}$ solution was added, and the mixture was extracted with EtOAc. The organic layer was washed with brine. The organic layer was dried and concentrated. The resulting residue was chromatographed on a silica gel column (hexane/EtOAc $=1 / 1)$ to give 9a $(2.59 \mathrm{~g}, 5.25 \mathrm{mmol}, 82 \%)$ as a pale yellow amorphous solid. ${ }^{1} \mathrm{H}-\mathrm{NMR}$ $\left(\mathrm{CDCl}_{3}\right) \delta: 1.48(9 \mathrm{H}, \mathrm{s}), 1.71-1.82(2 \mathrm{H}, \mathrm{m}), 1.85-1.98(2 \mathrm{H}, \mathrm{m}), 3.08(1 \mathrm{H}$ dd, $J=8.5,13.4 \mathrm{~Hz}), 3.29-3.42(2 \mathrm{H}, \mathrm{m}), 3.43(1 \mathrm{H}, \mathrm{dd}, J=4.0,13.4 \mathrm{~Hz})$ $3.60-3.70(2 \mathrm{H}, \mathrm{m}), 4.54-4.62(1 \mathrm{H}, \mathrm{m}), 4.65-4.73(1 \mathrm{H}, \mathrm{m}), 6.41(1 \mathrm{H}, \mathrm{dd}$ $J=5.8,16.4 \mathrm{~Hz}), 6.63(1 \mathrm{H}, \mathrm{d}, J=16.4 \mathrm{~Hz}), 6.84-6.90(2 \mathrm{H}, \mathrm{m}), 7.44(1 \mathrm{H}, \mathrm{t}$ $J=7.8 \mathrm{~Hz}), 7.53(1 \mathrm{H}, \mathrm{d}, J=7.8 \mathrm{~Hz}), 7.60(1 \mathrm{H}, \mathrm{d}, J=7.8 \mathrm{~Hz}), 7.65(1 \mathrm{H}, \mathrm{s})$, $8.08(1 \mathrm{H}, \mathrm{d}, J=9.6 \mathrm{~Hz})$

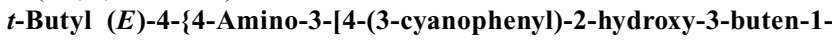
yl]phenoxy\}piperidine-1-carboxylate (10a) To a solution of $t$-butyl $(E)$ 4-\{3-[4-(3-cyanophenyl)-2-hydroxy-3-buten-1-yl]-4-nitrophenoxy\}piperidine-1-carboxylate 9a $(2.49 \mathrm{~g}, 5.05 \mathrm{mmol})$ in $\mathrm{AcOH}(25 \mathrm{ml})$ was added zinc powder $(1.98 \mathrm{~g}, 30.3 \mathrm{mmol})$ and the mixture was stirred at room temperature for $1 \mathrm{~h}$. The mixture was filtered, and the filtrate was concentrated. The resulting residue was diluted with EtOAc and washed with $\mathrm{NaHCO}_{3}$ solution and brine. The organic layer was dried and concentrated. The resulting residue was chromatographed on a silica gel column (hexane $/ \mathrm{EtOAc}=1 / 3$ ) to give $10 \mathrm{a}(1.39 \mathrm{~g}, 3.00 \mathrm{mmol}, 59 \%)$ as a brown oil. ${ }^{1} \mathrm{H}-\mathrm{NMR}\left(\mathrm{CDCl}_{3}\right) \delta$ $1.47(9 \mathrm{H}, \mathrm{s}), 1.62-1.74(2 \mathrm{H}, \mathrm{m}), 1.80-1.92(2 \mathrm{H}, \mathrm{m}), 2.82-2.87(2 \mathrm{H}, \mathrm{m})$, $3.21-3.32(2 \mathrm{H}, \mathrm{m}), 3.62-3.73(2 \mathrm{H}, \mathrm{m}), 4.23-4.30(1 \mathrm{H}, \mathrm{m}), 4.58-4.65$ $(1 \mathrm{H}, \mathrm{m}), 6.37(1 \mathrm{H}, \mathrm{dd}, J=5.9,15.7 \mathrm{~Hz}), 6.62(1 \mathrm{H}, \mathrm{d}, J=15.7 \mathrm{~Hz}), 6.67-$ $6.71(3 \mathrm{H}, \mathrm{m}), 7.42(1 \mathrm{H}, \mathrm{t}, J=7.8 \mathrm{~Hz}), 7.52(1 \mathrm{H}, \mathrm{d}, J=7.8 \mathrm{~Hz}), 7.58(1 \mathrm{H}, \mathrm{d}$, $J=7.8 \mathrm{~Hz}), 7.64(1 \mathrm{H}, \mathrm{s})$.

$t$-Butyl (E)-4-\{3-[4-(3-Cyanophenyl)-2-hydroxy-3-buten-1-yl]-4[(ethanesulfonyl)amino]phenoxy\}piperidine-1-carboxylate (11a) To a solution of $t$-butyl (E)-4-\{4-amino-3-[4-(3-cyanophenyl)-2-hydroxy-3-buten1-yl]phenoxy\}piperidine-1-carboxylate 10a $(1.38 \mathrm{~g}, 2.98 \mathrm{mmol})$ in $\mathrm{CH}_{2} \mathrm{Cl}_{2}$ $(15 \mathrm{ml})$ was added $\mathrm{EtSO}_{2} \mathrm{Cl}(0.340 \mathrm{ml}, 3.59 \mathrm{mmol})$ and pyridine $(0.290 \mathrm{ml}$, $3.59 \mathrm{mmol}$ ) and the mixture was stirred at room temperature for $4 \mathrm{~h} . \mathrm{H}_{2} \mathrm{O}$ was added, and the mixture was extracted with $\mathrm{CH}_{2} \mathrm{Cl}_{2}$. The organic layer was washed with brine. The organic layer was dried and concentrated. The resulting residue was chromatographed on a silica gel column (hexane/ EtOAc $=1 / 1)$ to give $11 \mathrm{a}(1.26 \mathrm{~g}, 2.27 \mathrm{mmol}, 76 \%)$ as a yellow oil. ${ }^{1} \mathrm{H}-\mathrm{NMR}$ 
$\left(\mathrm{CDCl}_{3}\right) \delta: 1.42(3 \mathrm{H}, \mathrm{t}, J=7.4 \mathrm{~Hz}), 1.47(9 \mathrm{H}, \mathrm{s}), 1.64-1.77(2 \mathrm{H}, \mathrm{m}), 1.81-$ $1.94(2 \mathrm{H}, \mathrm{m}), 2.98(2 \mathrm{H}, \mathrm{d}, J=6.3 \mathrm{~Hz}), 3.12(2 \mathrm{H}, \mathrm{q}, J=7.4 \mathrm{~Hz}), 3.24-3.36$ $(2 \mathrm{H}, \mathrm{m}), 3.58-3.70(2 \mathrm{H}, \mathrm{m}), 4.35-4.44(1 \mathrm{H}, \mathrm{m}), 4.59-4.67(1 \mathrm{H}, \mathrm{m}), 6.34$ $(1 \mathrm{H}, \mathrm{dd}, J=6.4,16.0 \mathrm{~Hz}), 6.62(1 \mathrm{H}, \mathrm{d}, J=16.0 \mathrm{~Hz}), 6.75(1 \mathrm{H}, \mathrm{d}, J=2.9 \mathrm{~Hz})$, $6.81(1 \mathrm{H}, \mathrm{dd}, J=2.9,8.8 \mathrm{~Hz}), 7.40(1 \mathrm{H}, \mathrm{d}, J=8.8 \mathrm{~Hz}), 7.44(1 \mathrm{H}, \mathrm{t}, J=7.8 \mathrm{~Hz})$, $7.55(1 \mathrm{H}, \mathrm{d}, J=7.8 \mathrm{~Hz}), 7.58(1 \mathrm{H}, \mathrm{d}, J=7.8 \mathrm{~Hz}), 7.63(1 \mathrm{H}, \mathrm{s})$

(E)-5-[1-(t-Butoxycarbonyl)piperidin-4-yloxy]-2-[2-(3-cyanophenyl)ethen-1-yll-1-(ethanesulfonyl)indoline (12a) To a solution of $t$-butyl $(E)$ 4-\{3-[4-(3-cyanophenyl)-2-hydroxy-3-buten-1-yl]-4-[(ethanesulfonyl)amino]phenoxy piperidine-1-carboxylate 11a $(1.20 \mathrm{~g}, 2.16 \mathrm{mmol})$ in THF $(30 \mathrm{ml})$ was added $n$-Bu $\mathrm{P}(0.770 \mathrm{ml}, 3.09 \mathrm{mmol})$ and ADDP $(0.710 \mathrm{~g}$, $2.81 \mathrm{mmol})$ in THF $(10 \mathrm{ml})$ and the mixture was stirred at room temperature for $3 \mathrm{~h}$. $\mathrm{NH}_{4} \mathrm{Cl}$ solution was added, and the mixture was extracted with EtOAc. The organic layer was washed with brine. The organic layer was dried and concentrated. The resulting residue was chromatographed on a silica gel column (hexane/EtOAc $=11 / 9)$ to give 12a $(1.05 \mathrm{~g}, 1.95 \mathrm{mmol}, 90 \%)$ as a colorless amorphous solid. ${ }^{1} \mathrm{H}-\mathrm{NMR}\left(\mathrm{CDCl}_{3}\right) \delta: 1.35(3 \mathrm{H}, \mathrm{t}, J=7.4 \mathrm{~Hz})$ $1.47(9 \mathrm{H}, \mathrm{s}), 1.67-1.78(2 \mathrm{H}, \mathrm{m}), 1.84-1.96(2 \mathrm{H}, \mathrm{m}), 2.89(1 \mathrm{H}, \mathrm{dd}, J=2.8$ $16.5 \mathrm{~Hz}), 2.98-3.13(2 \mathrm{H}, \mathrm{m}), 3.27-3.36(2 \mathrm{H}, \mathrm{m}), 3.58(1 \mathrm{H}, \mathrm{dd}, J=9.8$ $16.5 \mathrm{~Hz}), 3.64-3.74(2 \mathrm{H}, \mathrm{m}), 4.34-4.42(1 \mathrm{H}, \mathrm{m}), 5.05-5.12(1 \mathrm{H}, \mathrm{m})$ $6.30(1 \mathrm{H}, \mathrm{dd}, J=6.9,15.6 \mathrm{~Hz}), 6.69(1 \mathrm{H}, \mathrm{d}, J=15.6 \mathrm{~Hz}), 6.75-6.82(2 \mathrm{H}$ m), $7.33(1 \mathrm{H}, \mathrm{d}, J=8.6 \mathrm{~Hz}), 7.40(1 \mathrm{H}, \mathrm{t}, J=7.8 \mathrm{~Hz}), 7.51(1 \mathrm{H}, \mathrm{d}, J=7.8 \mathrm{~Hz})$ $7.58(1 \mathrm{H}, \mathrm{d}, J=7.8 \mathrm{~Hz}), 7.64(1 \mathrm{H}, \mathrm{s})$.

(E)-5-[1-(Acetimidoyl)piperidin-4-yloxy]-2-[2-(3-amidinophenyl)ethen-1-yl]-1-(ethanesulfonyl)indoline Dihydrochloride (13a) $\mathrm{HCl}$ gas was bubbled through a solution of $(E)-5-[1-(t$-butoxycarbonyl)piperidin-4yloxy]-2-[2-(3-cyanophenyl)ethen-1-yl]-1-(ethanesulfonyl)indoline 12a (970 $\mathrm{mg}, 1.80 \mathrm{mmol})$ in $\mathrm{CH}_{2} \mathrm{Cl}_{2}(12 \mathrm{ml})$ and $\mathrm{EtOH}(12 \mathrm{ml})$ at $0{ }^{\circ} \mathrm{C}$. Then the mixture was stirred at room temperature for $7 \mathrm{~h}$ and concentrated. The resulting residue was dissolved in $\mathrm{EtOH}(21 \mathrm{ml})$ and the solution was treated with $\mathrm{NH}_{4} \mathrm{Cl}(174 \mathrm{mg}, 3.25 \mathrm{mmol})$ and $\mathrm{NH}_{3}$ solution $(0.360 \mathrm{ml}, 5.92 \mathrm{mmol})$. The mixture was allowed to stand overnight at room temperature and concentrated. The resulting residue was purified by reverse-phase HPLC (YMCpack ODS, YMC, $\left.\mathrm{H}_{2} \mathrm{O} / \mathrm{MeCN}=17 / 3\right)$ to give $(E)$-2-[2-(3-amidinophenyl)ethen-1-yl]-1-(ethanesulfonyl)-5-(piperidin-4-yloxy)indoline $(684 \mathrm{mg}, 1.50$ $\mathrm{mmol}, 83 \%)$ as an amorphous solid. This solid $(481 \mathrm{mg}, 1.06 \mathrm{mmol})$ was dissolved in EtOH $(12 \mathrm{ml})$ and treated with ethyl acetimidate hydrochloride $(288 \mathrm{mg}, 2.33 \mathrm{mmol})$ and $\mathrm{Et}_{3} \mathrm{~N}(0.490 \mathrm{ml}, 3.53 \mathrm{mmol})$. The mixture was stirred overnight at room temperature. Ethyl acetimidate hydrochloride $(65 \mathrm{mg}, 0.53 \mathrm{mmol})$ and $\mathrm{Et}_{3} \mathrm{~N}(0.074 \mathrm{ml}, 0.53 \mathrm{mmol})$ was added, and the mixture was stirred for $3 \mathrm{~h}$. The mixture was concentrated and the resulting residue was purified by reverse-phase HPLC (YMC-pack ODS, YMC, $\left.\mathrm{H}_{2} \mathrm{O} / \mathrm{MeCN}=4 / 1\right)$ to give the free base of 13a $(430 \mathrm{mg}, 0.867 \mathrm{mmol})$ as an amorphous solid. This solid was dissolved in $\mathrm{MeOH}(8 \mathrm{ml})$ and treated with $4 \mathrm{~N} \mathrm{HCl}$ in dioxane $(0.640 \mathrm{ml}, 2.56 \mathrm{mmol})$. The mixture was concentrated and the resulting residue was lyophilized to give 13a $(411 \mathrm{mg}, 0.723 \mathrm{mmol}$, $68 \%$ ) as a colorless amorphous solid

Other derivatives $(\mathbf{1 3 b}-\mathbf{e})$ were similarly prepared.

t-Butyl (E)-4-\{3-[4-(3-Cyanophenyl)-2-hydroxy-3-buten-1-yl]-4[(ethoxycarbonylmethlysulfonyl)amino]phenoxy\}piperidine-1-carboxylate (11f) To a solution of $t$-butyl (E)-4-\{4-amino-3-[4-(3-cyanophenyl)-2hydroxy-3-buten-1-yl]phenoxy\}piperidine-1-carboxylate 10a $(1.50 \mathrm{~g}, 3.24$ $\mathrm{mmol})$ in $\mathrm{CH}_{2} \mathrm{Cl}_{2}(25 \mathrm{ml})$ was added ethoxycarbonylmethylsulfonyl chloride $(664 \mathrm{mg}, 3.56 \mathrm{mmol})$ in $\mathrm{CH}_{2} \mathrm{Cl}_{2}(10 \mathrm{ml})$ at $-10^{\circ} \mathrm{C}$ and the mixture was stirred for $1 \mathrm{~h}$. Pyridine $(0.290 \mathrm{ml}, 3.59 \mathrm{mmol})$ was added, and the mixture was stirred for $1.5 \mathrm{~h}$. Ethoxycarbonylmethylsulfonyl chloride $(664 \mathrm{mg}$, $3.56 \mathrm{mmol})$ in $\mathrm{CH}_{2} \mathrm{Cl}_{2}(2 \mathrm{ml})$ was added, and the mixture was stirred for $0.5 \mathrm{~h} . \mathrm{NaHCO}_{3}$ solution was added, and the mixture was extracted with $\mathrm{CH}_{2} \mathrm{Cl}_{2}$. The organic layer was washed with brine. The organic layer was dried and concentrated. The resulting residue was chromatographed on a silica gel column (hexane/EtOAc $=1 / 1)$ to give $11 f(576 \mathrm{mg}, 0.938 \mathrm{mmol}, 29 \%)$ as a yellow oil. ${ }^{1} \mathrm{H}-\mathrm{NMR}\left(\mathrm{CDCl}_{3}\right) \delta: 1.31(3 \mathrm{H}, \mathrm{t}, J=7.1 \mathrm{~Hz}), 1.47(9 \mathrm{H}, \mathrm{s})$ $1.65-1.77(2 \mathrm{H}, \mathrm{m}), 1.81-1.95(2 \mathrm{H}, \mathrm{m}), 3.02-3.14(2 \mathrm{H}, \mathrm{m}), 3.25-3.36$ $(2 \mathrm{H}, \mathrm{m}), 3.59-3.72(2 \mathrm{H}, \mathrm{m}), 4.01(1 \mathrm{H}, \mathrm{d}, J=14.7 \mathrm{~Hz}), 4.05(1 \mathrm{H}, \mathrm{d}$ $J=14.7 \mathrm{~Hz}), 4.26(2 \mathrm{H}, \mathrm{q}, J=7.1 \mathrm{~Hz}), 4.38-4.46(1 \mathrm{H}, \mathrm{m}), 4.61-4.68(1 \mathrm{H}$, $\mathrm{m}), 6.36(1 \mathrm{H}, \mathrm{dd}, J=6.4,15.9 \mathrm{~Hz}), 6.63(1 \mathrm{H}, \mathrm{d}, J=15.9 \mathrm{~Hz}), 6.78(1 \mathrm{H}, \mathrm{d}$ $J=2.8 \mathrm{~Hz}), 6.81(1 \mathrm{H}, \mathrm{dd}, J=2.8,8.7 \mathrm{~Hz}), 7.44(1 \mathrm{H}, \mathrm{t}, J=7.8 \mathrm{~Hz}), 7.48(1 \mathrm{H}$, d, $J=8.7 \mathrm{~Hz}), 7.54(1 \mathrm{H}, \mathrm{d}, J=7.8 \mathrm{~Hz}), 7.58(1 \mathrm{H}, \mathrm{d}, J=7.8 \mathrm{~Hz}), 7.63(1 \mathrm{H}, \mathrm{s})$.

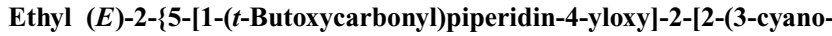
phenyl)ethen-1-yl]indolin-1-ylsulfonyl $\}$ acetate (12f) $t$-Butyl (E)-4-\{3[4-(3-cyanophenyl)-2-hydroxy-3-buten-1-yl]-4-[(ethoxycarbonylmethlysulfonyl)amino]phenoxy ; piperidine-1-carboxylate 11f was converted into $\mathbf{1 2 f}$ by the same procedure as that for 12a. 12f was obtained (86\%) as a pale yellow amorphous solid. ${ }^{1} \mathrm{H}-\mathrm{NMR}\left(\mathrm{CDCl}_{3}\right) \delta: 1.26(3 \mathrm{H}, \mathrm{t}, J=7.1 \mathrm{~Hz}), 1.47$
$(9 \mathrm{H}, \mathrm{s}), 1.67-1.80(2 \mathrm{H}, \mathrm{m}), 1.84-1.96(2 \mathrm{H}, \mathrm{m}), 2.86(1 \mathrm{H}, \mathrm{dd}, J=2.0$, $16.1 \mathrm{~Hz}), 3.27-3.37(2 \mathrm{H}, \mathrm{m}), 3.63-3.78(3 \mathrm{H}, \mathrm{m}), 3.99(1 \mathrm{H}, \mathrm{d}, J=14.2 \mathrm{~Hz})$ $4.03(1 \mathrm{H}, \mathrm{d}, J=14.2 \mathrm{~Hz}), 4.10-4.22(2 \mathrm{H}, \mathrm{m}), 4.35-4.43(1 \mathrm{H}, \mathrm{m}), 5.18$ $5.25(1 \mathrm{H}, \mathrm{m}), 6.31(1 \mathrm{H}, \mathrm{dd}, J=6.8,15.7 \mathrm{~Hz}), 6.67(1 \mathrm{H}, \mathrm{d}, J=15.7 \mathrm{~Hz}), 6.77$ $(1 \mathrm{H}, \mathrm{dd}, J=2.5,8.7 \mathrm{~Hz}), 6.81(1 \mathrm{H}, \mathrm{d}, J=2.5 \mathrm{~Hz}), 7.35(1 \mathrm{H}, \mathrm{d}, J=8.7 \mathrm{~Hz})$ $7.40(1 \mathrm{H}, \mathrm{t}, J=7.8 \mathrm{~Hz}), 7.51(1 \mathrm{H}, \mathrm{d}, J=7.8 \mathrm{~Hz}), 7.58(1 \mathrm{H}, \mathrm{d}, J=7.8 \mathrm{~Hz}), 7.64$ $(1 \mathrm{H}, \mathrm{s})$.

Other derivatives $(\mathbf{1 2 g}, \mathbf{1 2 h})$ were similarly prepared

12g: ${ }^{1} \mathrm{H}-\mathrm{NMR}\left(\mathrm{CDCl}_{3}\right) \delta: 1.47(9 \mathrm{H}, \mathrm{s}), 1.68-1.79(2 \mathrm{H}, \mathrm{m}), 1.83-1.96$ $(2 \mathrm{H}, \mathrm{m}), 2.24(3 \mathrm{H}, \mathrm{s}), 2.88(1 \mathrm{H}, \mathrm{d}, J=16.5 \mathrm{~Hz}), 3.27-3.37(2 \mathrm{H}, \mathrm{m}), 3.56$ $3.74(3 \mathrm{H}, \mathrm{m}), 4.35-4.43(1 \mathrm{H}, \mathrm{m}), 4.96-5.05(1 \mathrm{H}, \mathrm{m}), 6.28(1 \mathrm{H}, \mathrm{dd}$ $J=6.2,15.9 \mathrm{~Hz}), 6.47(1 \mathrm{H}, \mathrm{d}, J=15.9 \mathrm{~Hz}), 6.76(1 \mathrm{H}, \mathrm{s}), 6.79(1 \mathrm{H}, \mathrm{d}$ $J=8.7 \mathrm{~Hz}), 7.41(1 \mathrm{H}, \mathrm{t}, J=7.7 \mathrm{~Hz}), 7.53(2 \mathrm{H}, \mathrm{t}, J=7.7 \mathrm{~Hz}), 7.62(1 \mathrm{H}, \mathrm{s})$, $8.14(1 \mathrm{H}, \mathrm{d}, J=8.7 \mathrm{~Hz})$

12h: ${ }^{1} \mathrm{H}-\mathrm{NMR}\left(\mathrm{CDCl}_{3}\right) \delta: 1.47(9 \mathrm{H}, \mathrm{s}), 1.66-1.79(2 \mathrm{H}, \mathrm{m}), 1.83-1.97$ $(2 \mathrm{H}, \mathrm{m}), 2.20(3 \mathrm{H}, \mathrm{s}), 2.92(1 \mathrm{H}, \mathrm{d}, J=16.1 \mathrm{~Hz}), 3.25-3.38(2 \mathrm{H}, \mathrm{m}), 3.54$ $3.75(3 \mathrm{H}, \mathrm{m}), 4.36-4.45(1 \mathrm{H}, \mathrm{m}), 4.69(1 \mathrm{H}, \mathrm{d}, J=14.6 \mathrm{~Hz}), 4.90(1 \mathrm{H}, \mathrm{d}$ $J=14.6 \mathrm{~Hz}), 4.98-5.11(1 \mathrm{H}, \mathrm{m}), 6.28(1 \mathrm{H}, \mathrm{dd}, J=5.6,15.8 \mathrm{~Hz}), 6.55(1 \mathrm{H}$, d, $J=15.8 \mathrm{~Hz}), 6.75-6.82(2 \mathrm{H}, \mathrm{m}), 7.41(1 \mathrm{H}, \mathrm{t}, J=7.7 \mathrm{~Hz}), 7.53(1 \mathrm{H}, \mathrm{d}$, $J=7.7 \mathrm{~Hz}), 7.56(1 \mathrm{H}, \mathrm{d}, J=7.7 \mathrm{~Hz}), 7.63(1 \mathrm{H}, \mathrm{s}), 8.08-8.17(1 \mathrm{H}, \mathrm{m})$

(E)-5-[1-(t-Butoxycarbonyl)piperidin-4-yloxy]-2-[2-(3-cyanophenyl)ethen-1-yl]-1-(hydroxyacetyl)indoline (12i) To a solution of $(E)-1$ (acetoxyacetyl)-5-[1-( $t$-butoxycarbonyl)piperidin-4-yloxy]-2-[2-(3cyanophenyl)ethen-1-yl]indoline $\mathbf{1 2 h}(813 \mathrm{mg}, 1.49 \mathrm{mmol})$ in $\mathrm{MeOH}(12 \mathrm{ml})$ was added $\mathrm{K}_{2} \mathrm{CO}_{3}(51 \mathrm{mg}, 0.37 \mathrm{mmol})$ at $0{ }^{\circ} \mathrm{C}$. The mixture was stirred at room temperature for $1 \mathrm{~h}$. The mixture was concentrated and the resulting residue was diluted with EtOAc. The organic layer was washed with $\mathrm{H}_{2} \mathrm{O}$, brine. The organic layer was dried and concentrated. The resulting residue was chromatographed on a silica gel column (hexane/EtOAc $=2 / 3$ ) to give $12 \mathbf{i}(478 \mathrm{mg}, 0.949 \mathrm{mmol}, 64 \%)$ as a pale yellow oil. ${ }^{1} \mathrm{H}-\mathrm{NMR}\left(\mathrm{CDCl}_{3}\right) \delta$ $1.47(9 \mathrm{H}, \mathrm{s}), 1.68-1.80(2 \mathrm{H}, \mathrm{m}), 1.86-1.97(2 \mathrm{H}, \mathrm{m}), 2.92(1 \mathrm{H}, \mathrm{d}$ $J=15.8 \mathrm{~Hz}), 3.28-3.38(2 \mathrm{H}, \mathrm{m}), 3.55-3.75(3 \mathrm{H}, \mathrm{m}), 4.20-4.27(1 \mathrm{H}, \mathrm{m})$ $4.30-4.46(2 \mathrm{H}, \mathrm{m}), 4.84-4.92(1 \mathrm{H}, \mathrm{m}), 6.24(1 \mathrm{H}, \mathrm{dd}, J=7.1,15.9 \mathrm{~Hz})$ $6.48(1 \mathrm{H}, \mathrm{d}, J=15.9 \mathrm{~Hz}), 6.77-6.88(2 \mathrm{H}, \mathrm{m}), 7.42(1 \mathrm{H}, \mathrm{t}, J=8.0 \mathrm{~Hz}), 7.54$ $(2 \mathrm{H}, \mathrm{d}, J=8.0 \mathrm{~Hz}), 7.61(1 \mathrm{H}, \mathrm{s}), 8.13(1 \mathrm{H}, \mathrm{d}, J=8.5 \mathrm{~Hz})$.

Ethyl (E)-2-\{5-[1-(Acetimidoyl)piperidin-4-yloxy]-2-[2-(3-amidinophenyl)ethen-1-yl]indolin-1-ylsulfonyl $\}$ acetate Dihydrochloride (13f) Ethyl $(E)-2-\{5-[1-(t$-buthoxycarbonyl)piperidin-4-yloxy]-2-[2-(3-cyanophenyl)ethen-1-yl]indolin-1-ylsulfonyl $\}$ acetate $\mathbf{1 2 f}$ was converted into $\mathbf{1 3 f}$ by the similar procedure as that for 13a. 13f was obtained (61\%, 3 steps) as a colorless amorphous solid.

Other derivatives $(\mathbf{1 3 g}, \mathbf{1 3 i})$ were similarly prepared.

(E)-2-\{5-[1-(Acetimidoyl)piperidin-4-yloxy]-2-[2-(3-amidinophenyl)ethen-1-yl]indolin-1-ylsulfonyl $\}$ acetic Acid Dihydrochloride (13j) A solution of ethyl (E)-2-\{5-[1-(acetimidoyl)piperidin-4-yloxy]-2-[2-(3-amidinophenyl)ethen-1-yl]indolin-1-ylsulfonyl $\}$ acetate dihydrochloride $\mathbf{1 3 f}(248 \mathrm{mg}$, $0.448 \mathrm{mmol})$ in $4 \mathrm{~N} \mathrm{HCl}(12 \mathrm{ml})$ was stirred at $80^{\circ} \mathrm{C}$ for $1.5 \mathrm{~h}$. The mixture was concentrated and the resulting residue was purified by reverse-phase HPLC (YMC-pack ODS, YMC, $\mathrm{H}_{2} \mathrm{O} / \mathrm{MeCN}=87 / 13$ ) to give the free base of 13j as an amorphous solid. This solid was dissolved in $1 \mathrm{~N} \mathrm{HCl}(7 \mathrm{ml})$ and the mixture was concentrated. The resulting residue was lyophilized to give 13j (226 mg, $0.378 \mathrm{mmol}, 84 \%$ ) as a colorless amorphous solid.

$t$-Butyl 4-[2-Chloro-4-nitro-5-(trimethylsilylmethyl)phenoxy]piperidine-1-carboxylate (8k) and $t$-Butyl 4-[2-Chloro-4-nitro-3-(trimethylsilylmethyl)phenoxylpiperidine-1-carboxylate (8I) To a solution of 2 chloro-4-nitrophenol $14\left(\mathrm{R}^{4}=\mathrm{Cl}\right)(2.36 \mathrm{~g}, 13.6 \mathrm{mmol}), t$-butyl 4-hydroxypiperidine-1-carboxylate $15(3.32 \mathrm{~g}, 16.5 \mathrm{mmol})$ and $\mathrm{PPh}_{3}(5.11 \mathrm{~g}, 19.5 \mathrm{mmol})$ in $\mathrm{CH}_{2} \mathrm{Cl}_{2}(60 \mathrm{ml})$ was added DEAD $(3.10 \mathrm{ml}, 19.7 \mathrm{mmol})$ at $0{ }^{\circ} \mathrm{C}$ and the mixture was stirred at room temperature for $18 \mathrm{~h}$. The mixture was concentrated and the resulting residue was chromatographed on a silica gel column (hexane/EtOAc $=5 / 2)$ to give $t$-butyl 4-(2-chloro-4-nitro)phenoxypiperidine1-carboxylate $(3.90 \mathrm{~g}, 10.9 \mathrm{mmol})$ as a pale yellow solid. This solid $(12.90 \mathrm{~g}$, $36.2 \mathrm{mmol}$ ) was dissolved in THF $(200 \mathrm{ml})$ and treated with (trimethylsilylmethyl)magnesium chloride $\left(1.0 \mathrm{M}\right.$ in $\left.\mathrm{Et}_{2} \mathrm{O}, 40.0 \mathrm{ml}, 40.0 \mathrm{mmol}\right)$ slowly at $-25^{\circ} \mathrm{C}$ and the mixture was stirred at $-10{ }^{\circ} \mathrm{C}$ for $1 \mathrm{~h}$. DDQ $(10.21 \mathrm{~g}$, $45.0 \mathrm{mmol})$ in THF $(30 \mathrm{ml})$ was added slowly, and the mixture was stirred at $-10^{\circ} \mathrm{C}$ for $2 \mathrm{~h}$. $\mathrm{NH}_{4} \mathrm{Cl}$ solution was added, and the mixture was extracted with EtOAc. The organic layer was washed with brine. The organic layer was dried and concentrated. The resulting residue was chromatographed on a silica gel column (hexane/EtOAc $=4 / 1)$ to give $8 \mathbf{k}(4.40 \mathrm{~g}, 9.93 \mathrm{mmol}$, $22 \%)$ as a yellow oil and $\mathbf{8 1}(7.04 \mathrm{~g}, 15.9 \mathrm{mmol}, 35 \%)$ as a yellow oil.

8k: ${ }^{1} \mathrm{H}-\mathrm{NMR}\left(\mathrm{CDCl}_{3}\right) \delta$ : $0.02(9 \mathrm{H}, \mathrm{s}), 1.48(9 \mathrm{H}, \mathrm{s}), 1.79-1.98(4 \mathrm{H}, \mathrm{m})$, $2.64(2 \mathrm{H}, \mathrm{s}), 3.43-3.65(4 \mathrm{H}, \mathrm{m}), 4.60-4.68(1 \mathrm{H}, \mathrm{m}), 6.56(1 \mathrm{H}, \mathrm{s}), 8.16$ $(1 \mathrm{H}, \mathrm{s})$. 
81: ${ }^{1} \mathrm{H}-\mathrm{NMR}\left(\mathrm{CDCl}_{3}\right) \delta$ : $0.06(9 \mathrm{H}, \mathrm{s}), 1.48(9 \mathrm{H}, \mathrm{s}), 1.82-1.98(4 \mathrm{H}, \mathrm{m})$, $2.84(2 \mathrm{H}, \mathrm{s}), 3.48-3.66(4 \mathrm{H}, \mathrm{m}), 4.64-4.75(1 \mathrm{H}, \mathrm{m}), 6.76(1 \mathrm{H}, \mathrm{d}$, $J=9.2 \mathrm{~Hz}), 7.91(1 \mathrm{H}, \mathrm{d}, J=9.2 \mathrm{~Hz})$.

Ethyl $(E)-2-\{5-[1-(t-B u t o x y c a r b o n y l) p i p e r i d i n-4-y l o x y]-6-c h l o r o-2-[2-$ (3-cyanophenyl)ethen-1-yl]indolin-1-ylsulfonyl acetate (12k) $t$-Butyl 4[2-chloro-4-nitro-5-(trimethylsilylmethyl)phenoxy]piperidine-1-carboxylate $\mathbf{8 k}$ was converted into $\mathbf{1 2} \mathbf{k}$ by the same procedure as that for $\mathbf{1 2} \mathbf{a}$. $\mathbf{1 2} \mathbf{k}$ was obtained ( $8 \%, 4$ steps) as a pale yellow oil. ${ }^{1} \mathrm{H}-\mathrm{NMR}\left(\mathrm{CDCl}_{3}\right) \delta: 1.28(3 \mathrm{H}, \mathrm{t}$, $J=7.4 \mathrm{~Hz}), 1.47(9 \mathrm{H}, \mathrm{s}), 1.76-1.95(4 \mathrm{H}, \mathrm{m}), 2.85(1 \mathrm{H}, \mathrm{dd}, J=1.8,16.2 \mathrm{~Hz})$, $3.36-3.47(2 \mathrm{H}, \mathrm{m}), 3.63-3.77(3 \mathrm{H}, \mathrm{m}), 4.05(2 \mathrm{H}, \mathrm{s}), 4.10-4.22(2 \mathrm{H}, \mathrm{m})$, $4.39-4.48(1 \mathrm{H}, \mathrm{m}), 5.19-5.27(1 \mathrm{H}, \mathrm{m}), 6.30(1 \mathrm{H}, \mathrm{dd}, J=6.8,15.4 \mathrm{~Hz})$, $6.67(1 \mathrm{H}, \mathrm{d}, J=15.4 \mathrm{~Hz}), 6.87(1 \mathrm{H}, \mathrm{s}), 7.41(1 \mathrm{H}, \mathrm{t}, J=7.8 \mathrm{~Hz}), 7.49(1 \mathrm{H}, \mathrm{s})$, $7.52(1 \mathrm{H}, \mathrm{d}, J=7.8 \mathrm{~Hz}), 7.58(1 \mathrm{H}, \mathrm{d}, J=7.8 \mathrm{~Hz}), 7.65(1 \mathrm{H}, \mathrm{s})$

Other derivatives $(\mathbf{1 2 l}-\mathbf{s})$ were similarly prepared

Ethyl $(E)-2-\{5-[1-(t-B u t o x y c a r b o n y l) p i p e r i d i n-4-y l o x y]-6-c a r b o x y-2-$ [2-(3-cyanophenyl)ethen-1-yl]indolin-1-ylsulfonyl\}acetate (12t) To a solution of ethyl (E)-2-\{5-[1-(t-butoxycarbonyl)piperidin-4-yloxy]-2-[2-(3cyanophenyl)ethen-1-yl]-6-(1,3-dioxolan-2-yl)indolin-1-ylsulfonyl $\}$ acetate $12 \mathrm{~s}(850 \mathrm{mg}, 1.27 \mathrm{mmol})$ in acetone $(40 \mathrm{ml})$ and $\mathrm{H}_{2} \mathrm{O}(10 \mathrm{ml})$ was added $p$ TsOH monohydrate $(80 \mathrm{mg}, 0.42 \mathrm{mmol})$ and the mixture was stirred at room temperature for $30 \mathrm{~min}$. $\mathrm{NaHCO}_{3}$ solution was added, and the mixture was concentrated. The resulting residue was extracted with EtOAc and the organic layer was washed with brine. The organic layer was dried and concentrated to give Ethyl (E)-2-\{5-[1-(t-butoxycarbonyl)piperidin-4-yloxy]-2-[2(3-cyanophenyl)ethen-1-yl]-6-formylindolin-1-ylsulfonyl \}acetate $\quad(780 \mathrm{mg})$ as a yellow amorphous solid. This solid $(770 \mathrm{mg}, 1.23 \mathrm{mmol})$ and 2-methyl2-butene $(8.00 \mathrm{ml}, 75.5 \mathrm{mmol})$ was dissolved in $t$-BuOH $(80 \mathrm{ml})$ and treated with $\mathrm{NaClO}_{2}(1.03 \mathrm{~g}, 11.4 \mathrm{mmol})$ and $\mathrm{NaH}_{2} \mathrm{PO}_{4}(1.37 \mathrm{~g}, 11.4 \mathrm{mmol})$ in $\mathrm{H}_{2} \mathrm{O}$ $(12 \mathrm{ml})$ slowly. The mixture was stirred at room temperature for $0.5 \mathrm{~h}$ and concentrated. The resulting residue was extracted with EtOAc. The organic layer was washed with $\mathrm{NaHCO}_{3}$ solution and brine. The organic layer was dried and concentrated to give $\mathbf{1 2 t}(920 \mathrm{mg}$, quant.) as a yellow amorphous solid. ${ }^{1} \mathrm{H}-\mathrm{NMR}\left(\mathrm{CDCl}_{3}\right) \delta: 1.29(3 \mathrm{H}, \mathrm{t}, J=7.2 \mathrm{~Hz}), 1.47(9 \mathrm{H}, \mathrm{s}), 1.81-1.86$ $(2 \mathrm{H}, \mathrm{m}), 2.04-2.11(2 \mathrm{H}, \mathrm{m}), 2.94(1 \mathrm{H}, \mathrm{dd}, J=2.3,17.0 \mathrm{~Hz}), 3.26(2 \mathrm{H}, \mathrm{t}$ $J=10.3 \mathrm{~Hz}), 3.79-3.86(3 \mathrm{H}, \mathrm{m}), 4.07(2 \mathrm{H}, \mathrm{s}), 4.11-4.24(2 \mathrm{H}, \mathrm{m}), 4.66$ $4.68(1 \mathrm{H}, \mathrm{m}), 5.28(1 \mathrm{H}, \mathrm{t}, J=6.8 \mathrm{~Hz}), 6.30(1 \mathrm{H}, \mathrm{dd}, J=6.8,15.7 \mathrm{~Hz}), 6.68$ $(1 \mathrm{H}, \mathrm{d}, J=15.7 \mathrm{~Hz}), 6.99(1 \mathrm{H}, \mathrm{s}), 7.41(1 \mathrm{H}, \mathrm{t}, J=7.7 \mathrm{~Hz}), 7.52(1 \mathrm{H}, \mathrm{d}$, $J=7.7 \mathrm{~Hz}), 7.58(1 \mathrm{H}, \mathrm{d}, J=7.7 \mathrm{~Hz}), 7.65(1 \mathrm{H}, \mathrm{s}), 8.13(1 \mathrm{H}, \mathrm{s})$.

Ethyl $(E)-2-\{5-[1-(t$-Butoxycarbonyl)piperidin-4-yloxy]-6-carbamoyl2-[2-(3-cyanophenyl)ethen-1-yl]indolin-1-ylsulfonyl\}acetate (12u) To a solution of ethyl (E)-2-\{5-[1-(t-butoxycarbonyl)piperidin-4-yloxy]-6-carboxy-2-[2-(3-cyanophenyl)ethen-1-yl]indolin-1-ylsulfonyl $\}$ acetate 12t (460 $\mathrm{mg}, 0.719 \mathrm{mmol})$ in $\mathrm{CH}_{2} \mathrm{Cl}_{2}(10 \mathrm{ml})$ was added $\mathrm{Et}_{3} \mathrm{~N}(0.120 \mathrm{ml}, 0.866 \mathrm{mmol})$ and $\mathrm{ClCO}_{2} i-\operatorname{Pr}(0.110 \mathrm{ml}, 0.966 \mathrm{mmol})$ and the mixture was stirred at room temperature for $30 \mathrm{~min}$. $\mathrm{NH}_{3}$ solution $(0.090 \mathrm{ml}, 1.5 \mathrm{mmol})$ was added, and the mixture was stirred at room temperature for $30 \mathrm{~min}$. The mixture was extracted with EtOAc and the organic layer was washed with $\mathrm{H}_{2} \mathrm{O}$ and brine. The organic layer was dried and concentrated. The resulting residue was chromatographed on a silica gel column (EtOAc) to give 12u (380 mg, $0.595 \mathrm{mmol}, 83 \%)$ as a brown amorphous solid. ${ }^{1} \mathrm{H}-\mathrm{NMR}\left(\mathrm{CDCl}_{3}\right) \delta: 1.29$ $(3 \mathrm{H}, \mathrm{t}, J=7.2 \mathrm{~Hz}), 1.47(9 \mathrm{H}, \mathrm{s}), 1.73-1.83(2 \mathrm{H}, \mathrm{m}), 1.99-2.08(2 \mathrm{H}, \mathrm{m})$, $2.90(1 \mathrm{H}, \mathrm{dd}, J=2.4,16.8 \mathrm{~Hz}), 3.25(2 \mathrm{H}, \mathrm{t}, J=10.5 \mathrm{~Hz}), 3.77-3.84(3 \mathrm{H}, \mathrm{m})$ $4.05(1 \mathrm{H}, \mathrm{d}, J=14.4 \mathrm{~Hz}), 4.08(1 \mathrm{H}, \mathrm{d}, J=14.4 \mathrm{~Hz}), 4.10-4.24(2 \mathrm{H}, \mathrm{m})$, $4.55-4.59(1 \mathrm{H}, \mathrm{m}), 5.27(1 \mathrm{H}, \mathrm{t}, J=7.0 \mathrm{~Hz}), 6.29(1 \mathrm{H}, \mathrm{dd}, J=6.6,15.7 \mathrm{~Hz})$ $6.67(1 \mathrm{H}, \mathrm{d}, J=15.7 \mathrm{~Hz}), 6.92(1 \mathrm{H}, \mathrm{s}), 7.40(1 \mathrm{H}, \mathrm{t}, J=7.8 \mathrm{~Hz}), 7.51(1 \mathrm{H}, \mathrm{d}$, $J=7.8 \mathrm{~Hz}), 7.57(1 \mathrm{H}, \mathrm{d}, J=7.8 \mathrm{~Hz}), 7.65(1 \mathrm{H}, \mathrm{s}), 8.18(1 \mathrm{H}, \mathrm{s})$.

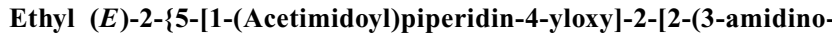
phenyl)ethen-1-yl]-6-chloroindolin-1-ylsulfonyl $\}$ acetate Dihydrochloride (13k) Ethyl (E)-2-\{5-[1-(t-butoxycarbonyl)piperidin-4-yloxy]-6-chloro-2[2-(3-cyanophenyl)ethen-1-yl]indolin-1-ylsulfonyl $\}$ acetate $\mathbf{1 2 k}$ was converted into $13 \mathbf{k}$ by the same procedure as that for 13a. 13k was obtained $(50 \%, 3$ steps $)$ as a colorless amorphous solid.

Other derivatives $(\mathbf{1 3 l}-\mathbf{r}, \mathbf{1 3 t}, \mathbf{1 3 \mathbf { u }})$ were similarly prepared

$t$-Butyl (E)-4-\{3-[4-(3-Cyanophenyl)-2-oxo-3-buten-1-yl]-4-nitrophenoxy\}piperidine-1-carboxylate (16) To a solution of $t$-butyl $(E)-4-\{3-[4-$ (3-cyanophenyl)-2-hydroxy-3-buten-1-yl]-4-nitrophenoxy piperidine-1-carboxylate $9 \mathrm{a}(2.55 \mathrm{~g}, 5.17 \mathrm{mmol})$ in $\mathrm{CH}_{2} \mathrm{Cl}_{2}(50 \mathrm{ml})$ was added pyridinium chlorochromate (PCC) $(1.45 \mathrm{~g}, 6.73 \mathrm{mmol})$ at $0{ }^{\circ} \mathrm{C}$ and the mixture was stirred overnight at room temperature. The mixture was filtered, and the filtrate was washed with $\mathrm{H}_{2} \mathrm{O}$ and brine. The organic layer was dried and concentrated. The resulting residue was chromatographed on a silica gel column $\left(\mathrm{CH}_{2} \mathrm{Cl}_{2} / \mathrm{EtOAc}=4 / 1\right)$ to give $16(1.79 \mathrm{~g}, 3.64 \mathrm{mmol}, 70 \%)$ as a colorless solid. ${ }^{1} \mathrm{H}-\mathrm{NMR}\left(\mathrm{CDCl}_{3}\right) \delta: 1.48(9 \mathrm{H}, \mathrm{s}), 1.73-1.86(2 \mathrm{H}, \mathrm{m}), 1.90-2.02$ $(2 \mathrm{H}, \mathrm{m}), 3.30-3.44(2 \mathrm{H}, \mathrm{m}), 3.61-3.75(2 \mathrm{H}, \mathrm{m}), 4.35(2 \mathrm{H}, \mathrm{s}), 4.57-4.64$
$(1 \mathrm{H}, \mathrm{m}), 6.80(1 \mathrm{H}, \mathrm{d}, J=2.7 \mathrm{~Hz}), 6.92(1 \mathrm{H}, \mathrm{dd}, J=2.7,9.2 \mathrm{~Hz}), 6.94(1 \mathrm{H}, \mathrm{d}$, $J=16.1 \mathrm{~Hz}), 7.54(1 \mathrm{H}, \mathrm{t}, J=7.9 \mathrm{~Hz}), 7.63(1 \mathrm{H}, \mathrm{d}, J=16.1 \mathrm{~Hz}), 7.69(1 \mathrm{H}, \mathrm{d}$, $J=7.9 \mathrm{~Hz}), 7.80(1 \mathrm{H}, \mathrm{d}, J=7.9 \mathrm{~Hz}), 7.86(1 \mathrm{H}, \mathrm{s}), 8.22(1 \mathrm{H}, \mathrm{d}, J=9.2 \mathrm{~Hz})$.

t-Butyl $(S)$-(E)-4-\{4-Amino-3-[4-(3-cyanophenyl)-2-hydroxy-3-buten1-yl]phenoxy\}piperidine-1-carboxylate $((S)-10 a)$ To a solution of $(R)$ $\alpha, \alpha$-diphenyl-2-pyrrolidinemethanol $(R)-17(665 \mathrm{mg}, 2.62 \mathrm{mmol})$ in THF $(30 \mathrm{ml})$ was added trimethoxyborane $(409 \mathrm{mg}, 3.94 \mathrm{mmol})$ in THF $(5 \mathrm{ml})$ and the mixture was stirred at room temperature for $1 \mathrm{~h}$. Borane-dimethylsulfide complex $(2.0 \mathrm{M}$ in THF, $2.62 \mathrm{ml}, 5.24 \mathrm{mmol})$ and $t$-butyl $(E)-4-\{3-[4-(3-$ cyanophenyl)-2-oxo-3-buten-1-yl]-4-nitrophenoxy \} piperidine-1-carboxylate $16(1.29 \mathrm{~g}, 2.62 \mathrm{mmol})$ in THF $(25 \mathrm{ml})$ was added, and the mixture was stirred at room temperature for $1.5 \mathrm{~h}$. $\mathrm{NH}_{4} \mathrm{Cl}$ solution was added, and the mixture was extracted with EtOAc. The organic layer was washed with brine. The organic layer was dried and concentrated. The resulting residue was chromatographed on a silica gel column (hexane/EtOAc $=1 / 1$ ) to give $t$ butyl $(S)-(E)-4-\{3-[4-(3-c y a n o p h e n y l)-2-h y d r o x y-3-b u t e n-1-y l]-4-n i t r o p h e-$ noxy piperidine-1-carboxylate $(963 \mathrm{mg}, 1.95 \mathrm{mmol}, 74 \%)$ as a colorless amorphous solid. To a solution of this solid in $\mathrm{AcOH}(15 \mathrm{ml})$ was added zinc powder $(762 \mathrm{mg}, 11.7 \mathrm{mmol})$ and the mixture was stirred at room temperature for $1 \mathrm{~h}$. Zinc powder $(762 \mathrm{mg}, 11.7 \mathrm{mmol})$ was added, and the mixture was stirred at room temperature for $1 \mathrm{~h}$. The mixture was filtered, and the filtrate was concentrated. The resulting residue was diluted with EtOAc and washed with $\mathrm{NaHCO}_{3}$ solution and brine. The organic layer was dried and concentrated. The resulting residue was chromatographed on a silica gel column (hexane/EtOAc $=1 / 3$ ) and then purified by chiral HPLC (Daicel Chiralcel OD, Daicel Chemical Industries Ltd., hexane $/ i-\mathrm{PrOH}=7 / 3)$ to give $(S)$ 10a (432 mg, $0.932 \mathrm{mmol}, 48 \%,>99 \%$ ee) as a yellow oil. $[\alpha]_{\mathrm{D}}+46.1^{\circ}(c=$ $0.43, \mathrm{MeOH})$.

$(R)-(E)-2-\{5-[1-($ Acetimidoyl)piperidin-4-yloxy]-2-[2-(3-amidinophenyl)ethen-1-yl]indolin-1-ylsulfonyl $\}$ acetic Acid Dihydrochloride $((R)$ 13j) $t$-Butyl $(S)$ - $(E)$-4-\{4-amino-3-[4-(3-cyanophenyl)-2-hydroxy-3-buten1-yl]phenoxy piperidine-1-carboxylate $(S)-\mathbf{1 0 a}$ was converted into $(R)-\mathbf{1 3} \mathbf{j}$ by the same procedure as that for racemate $\mathbf{1 3} \mathbf{j}$. $(R)-\mathbf{1 3} \mathbf{j}$ was obtained $(10 \%$, 6 steps $)$ as a colorless amorphous solid. $[\alpha]_{\mathrm{D}}-13.6^{\circ}(c=0.29, \mathrm{MeOH})$.

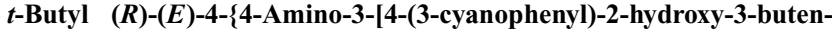
1-yl]phenoxy\}piperidine-1-carboxylate $((R)-10 a) \quad t$-Butyl $(E)-4-\{3-[4-(3-$ cyanophenyl)-2-oxo-3-buten-1-yl]-4-nitrophenoxy \}piperidine-1-carboxylate 16 was converted into $(R)-\mathbf{1 0 a}$ by the same procedure as that for $(S)-\mathbf{1 0 a}$. $(S)$ - $\alpha, \alpha$-Diphenyl-2-pyrrolidinemethanol $(S)$-17 was used instead of $(R)-17$ $(R)-10$ a was obtained $(17 \%, 2$ steps, $>99 \%$ ee $)$ as a brown amorphous solid. $[\alpha]_{\mathrm{D}}-43.6^{\circ}(c=1.02, \mathrm{MeOH})$.

$(S)-(E)-2-\{5-[1-($ Acetimidoyl)piperidin-4-yloxy]-2-[2-(3-amidinophenyl)ethen-1-yl]indolin-1-ylsulfonyl \}acetic Acid Dihydrochloride ( $(S)$ 13j) $t$-Butyl $(R)-(E)-4$-\{4-amino-3-[4-(3-cyanophenyl)-2-hydroxy-3-buten1-yl]phenoxy \}piperidine-1-carboxylate $(R)-\mathbf{1 0 a}$ was converted into $(S)-\mathbf{1 3} \mathbf{j}$ by the same procedure as that for racemate $\mathbf{1 3} \mathbf{j}$. $(S)-\mathbf{1 3} \mathbf{j}$ was obtained $(9 \%, 6$ steps $)$ as a yellow amorphous solid. $[\alpha]_{\mathrm{D}}+16.1^{\circ}(c=0.99, \mathrm{MeOH})$.

5-Cyano-2-(methoxymethoxy)cinnamaldehyde (20) To a solution of 5-cyano-2-hydroxybenzaldehyde $18(2.08 \mathrm{~g}, 14.1 \mathrm{mmol})$ in toluene $(60 \mathrm{ml})$ was added (triphenylphosphoranylidene)acetaldehyde $(4.52 \mathrm{~g}, 14.9 \mathrm{mmol})$ and the mixture was stirred at $70{ }^{\circ} \mathrm{C}$ for $1.5 \mathrm{~h}$. The mixture was concentrated and the resulting residue was chromatographed on a silica gel column (hexane/EtOAc = 1/1) to give 5-cyano-2-hydroxycinnamaldehyde 19 (1.65 g, $9.53 \mathrm{mmol})$ as a colorless solid. To a solution of this solid $(1.63 \mathrm{~g}$ $9.41 \mathrm{mmol})$ in DMA $(30 \mathrm{ml})$ was added methoxymethylchloride $(0.850 \mathrm{ml}$, $11.2 \mathrm{mmol})$ and $\mathrm{Et}_{3} \mathrm{~N}(1.57 \mathrm{ml}, 11.3 \mathrm{mmol})$ at $0{ }^{\circ} \mathrm{C}$ and the mixture was stirred at room temperature for $5 \mathrm{~h}$. Methoxymethylchloride $(0.210 \mathrm{mmol}$, $2.76 \mathrm{mmol})$ and $\mathrm{Et}_{3} \mathrm{~N}(0.390 \mathrm{ml}, 2.81 \mathrm{mmol})$ were added, and the mixture was stirred for $2 \mathrm{~h}$. Brine was added, and the mixture was extracted with EtOAc. The organic layer was washed with brine. The organic layer was dried and concentrated. The resulting residue was chromatographed on a silica gel column (hexane/EtOAc $=17 / 3)$ to give $20(1.86 \mathrm{~g}, 8.56 \mathrm{mmol}, 61 \%)$ as a colorless solid. ${ }^{1} \mathrm{H}-\mathrm{NMR}\left(\mathrm{CDCl}_{3}\right) \delta: 3.52(3 \mathrm{H}, \mathrm{s}), 5.36(2 \mathrm{H}, \mathrm{s}), 6.80$ $(1 \mathrm{H}, \mathrm{dd}, J=7.6,16.2 \mathrm{~Hz}), 7.30(1 \mathrm{H}, \mathrm{d}, J=8.7 \mathrm{~Hz}), 7.66(1 \mathrm{H}, \mathrm{dd}, J=2.1$, $8.7 \mathrm{~Hz}), 7.75(1 \mathrm{H}, \mathrm{d}, J=16.2 \mathrm{~Hz}), 7.84(1 \mathrm{H}, \mathrm{d}, J=2.1 \mathrm{~Hz}), 9.74(1 \mathrm{H}, \mathrm{d}$ $J=7.6 \mathrm{~Hz})$.

Ethyl $(E)-2-(5-[1-(t-B u t o x y c a r b o n y l) p i p e r i d i n-4-y l o x y]-2-\{2-[5-c y a n o-$ 2-(methoxymethoxy)phenyl]ethen-1-yl $\}$ indolin-1-ylsulfonyl)acetate (21c) 5-Cyano-2-(methoxymethoxy)cinnamaldehyde 20 was converted into $21 \mathrm{c}$ by the similar procedure as that for 12a. 21c was obtained $(15 \%, 4$ steps) as a colorless amorphous solid. ${ }^{1} \mathrm{H}-\mathrm{NMR}\left(\mathrm{CDCl}_{3}\right) \delta: 1.26(3 \mathrm{H}, \mathrm{t}, J=7.1 \mathrm{~Hz})$, $1.47(9 \mathrm{H}, \mathrm{s}), 1.68-1.79(2 \mathrm{H}, \mathrm{m}), 1.85-1.95(2 \mathrm{H}, \mathrm{m}), 2.87(1 \mathrm{H}, \mathrm{dd}, J=2.1$, $16.3 \mathrm{~Hz}), 3.28-3.38(2 \mathrm{H}, \mathrm{m}), 3.47(3 \mathrm{H}, \mathrm{s}), 3.64-3.77(3 \mathrm{H}, \mathrm{m}), 3.98(1 \mathrm{H}$, d, $J=14.1 \mathrm{~Hz}), 4.03(1 \mathrm{H}, \mathrm{d}, J=14.1 \mathrm{~Hz}), 4.09-4.20(2 \mathrm{H}, \mathrm{m}), 4.34-4.42$ 
$(1 \mathrm{H}, \mathrm{m}), 5.17-5.24(1 \mathrm{H}, \mathrm{m}), 5.25(2 \mathrm{H}, \mathrm{s}), 6.29(1 \mathrm{H}, \mathrm{dd}, J=7.4,15.9 \mathrm{~Hz})$, $6.77(1 \mathrm{H}, \mathrm{dd}, J=2.5,8.7 \mathrm{~Hz}), 6.81(1 \mathrm{H}, \mathrm{d}, J=2.5 \mathrm{~Hz}), 6.96(1 \mathrm{H}, \mathrm{d}$, $J=15.9 \mathrm{~Hz}), 7.14(1 \mathrm{H}, \mathrm{d}, J=8.6 \mathrm{~Hz}), 7.34(1 \mathrm{H}, \mathrm{d}, J=8.7 \mathrm{~Hz}), 7.47(1 \mathrm{H}, \mathrm{dd}$, $J=2.1,8.6 \mathrm{~Hz}), 7.69(1 \mathrm{H}, \mathrm{d}, J=2.1 \mathrm{~Hz})$.

Other derivatives (21a, 21d, 21e) were similarly prepared.

(E)-5-[1-(t-Butoxycarbonyl)piperidin-4-yloxy]-2-[2-(5-cyano-2methoxyphenyl)ethen-1-yl]-1-(ethanesulfonyl)indoline (21b) To a solution of $(E)-5-[1-(t$-butoxycarbonyl)piperidin-4-yloxy]-2-\{2-[5-cyano-2(methoxymethoxy)phenyl]ethen-1-yl\}-1-(ethanesulfonyl)indoline 21a (530 $\mathrm{mg}, 0.887 \mathrm{mmol})$ in EtOAc $(5 \mathrm{ml})$ was added $4 \mathrm{~N} \mathrm{HCl}$ in EtOAc $(5 \mathrm{ml})$ at $0{ }^{\circ} \mathrm{C}$ and the mixture was stirred at room temperature for $4 \mathrm{~h}$. The mixture was concentrated to give monohydrochloride of $(E)$-2-[2-(5-cyano-2hydroxyphenyl)ethen-1-yl]-1-(ethanesulfonyl)-5-(piperidin-4-yloxy)indoline $(514 \mathrm{mg})$ as a pale brown oil. To a solution of this oil $(514 \mathrm{mg})$ in acetone $(10 \mathrm{ml})$ and $\mathrm{H}_{2} \mathrm{O}(10 \mathrm{ml})$ was added $\mathrm{Boc}_{2} \mathrm{O}(203 \mathrm{mg}, 0.930 \mathrm{mmol})$ and $\mathrm{NaHCO}_{3}(82.0 \mathrm{mg}, 0.976 \mathrm{mmol})$ at $0{ }^{\circ} \mathrm{C}$ and the mixture was stirred at room temperature for $5 \mathrm{~h}$. The mixture was concentrated and the resulting residue was extracted with EtOAc. The organic layer was washed with $\mathrm{NH}_{4} \mathrm{Cl}$ solution and brine. The organic layer was dried and concentrated. The resulting residue was chromatographed on a silica gel column (hexane/EtOAc $=2 / 3$ ) to give $(E)-5$-[1-( $t$-butoxycarbonyl)piperidin-4-yloxy]-2-[2-(5-cyano-2-hydroxyphenyl)ethen-1-yl]-1-(ethanesulfonyl)indoline $(472 \mathrm{mg}, 0.852 \mathrm{mmol})$ as a colorless oil. To a solution of this oil $(452 \mathrm{mg}, 0.816 \mathrm{mmol})$ in benzene $(16 \mathrm{ml})$ and $\mathrm{MeOH}(4 \mathrm{ml})$ was added (trimethylsilyl)diazomethane $(2.0 \mathrm{M}$ in hexane, $1.22 \mathrm{ml}, 2.44 \mathrm{mmol}$ ) and the mixture was stirred at room temperature for $0.5 \mathrm{~h}$. The mixture was concentrated and the resulting residue was chromatographed on a silica gel column (hexane/EtOAc $=1 / 1$ ) to give $\mathbf{2 1 b}$ (448 mg, $0.789 \mathrm{mmol}, 93 \%)$ as a colorless oil. ${ }^{1} \mathrm{H}-\mathrm{NMR}\left(\mathrm{CDCl}_{3}\right) \delta: 1.36$ $(3 \mathrm{H}, \mathrm{t}, J=7.4 \mathrm{~Hz}), 1.47(9 \mathrm{H}, \mathrm{s}), 1.67-1.79(2 \mathrm{H}, \mathrm{m}), 1.83-1.96(2 \mathrm{H}, \mathrm{m})$, $2.89(1 \mathrm{H}, \mathrm{dd}, J=2.7,16.4 \mathrm{~Hz}), 2.98-3.14(2 \mathrm{H}, \mathrm{m}), 3.26-3.39(2 \mathrm{H}, \mathrm{m})$, $3.57(1 \mathrm{H}, \mathrm{dd}, J=9.7,16.4 \mathrm{~Hz}), 3.63-3.75(2 \mathrm{H}, \mathrm{m}), 3.89(3 \mathrm{H}, \mathrm{s}), 4.33-4.42$ $(1 \mathrm{H}, \mathrm{m}), 5.04-5.12(1 \mathrm{H}, \mathrm{m}), 6.30(1 \mathrm{H}, \mathrm{dd}, J=7.5,16.0 \mathrm{~Hz}), 6.76(1 \mathrm{H}, \mathrm{dd}$ $J=2.4,8.7 \mathrm{~Hz}), 6.79(1 \mathrm{H}, \mathrm{d}, J=2.4 \mathrm{~Hz}), 6.90(1 \mathrm{H}, \mathrm{d}, J=8.6 \mathrm{~Hz}), 6.93(1 \mathrm{H}, \mathrm{d}$, $J=16.0 \mathrm{~Hz}), 7.31(1 \mathrm{H}, \mathrm{d}, J=8.7 \mathrm{~Hz}), 7.51(1 \mathrm{H}, \mathrm{dd}, J=2.0,8.6 \mathrm{~Hz}), 7.65(1 \mathrm{H}$, d, $J=2.0 \mathrm{~Hz}$.

Ethyl (E)-2-\{5-[1-(Acetimidoyl)piperidin-4-yloxy]-2-[2-(5-amidino-2hydroxyphenyl)ethen-1-yl]indolin-1-ylsulfonyl \}acetate Dihydrochloride (22c) Ethyl (E)-2-(5-[1-(t-butoxycarbonyl)piperidin-4-yloxy]-2-\{2-[5cyano-2-(methoxymethoxy)phenyl]ethen-1-yl \}indolin-1-ylsulfonyl)acetate 21c was converted into 22c by the similar procedure as that for 13a. 22c was obtained ( $23 \%, 3$ steps) as a pink amorphous solid.

Other derivatives (22a, 22b, 22d, 22e) were similarly prepared.

$(E)-2-\{5-[1-($ Acetimidoyl)piperidin-4-yloxy]-2-[2-(5-amidino-2hydroxyphenyl)ethen-1-yl]indolin-1-ylsulfonyl $\}$ acetic Acid Dihydrochloride (22f) A solution of dihydrochloride of ethyl $(E)-2-\{5-[1-$ (acetimidoyl)piperidin-4-yloxy]-2-[2-(5-amidino-2-hydroxyphenyl)ethen-1-yl]indolin-1-ylsulfonyl \}acetate $22 \mathrm{c}(223 \mathrm{mg}, 0.347 \mathrm{mmol})$ in $1 \mathrm{~N} \mathrm{HCl}(10 \mathrm{ml})$ was stirred at $80^{\circ} \mathrm{C}$ for $7.5 \mathrm{~h}$. The mixture was concentrated and the resulting residue was purified by reverse-phase HPLC (YMC-pack ODS, YMC, $\left.\mathrm{H}_{2} \mathrm{O} / \mathrm{MeCN}=9 / 1\right)$ to give the free base of $22 \mathbf{f}$ as an amorphous solid. This solid was dissolved in $1 \mathrm{~N} \mathrm{HCl}(10 \mathrm{ml})$ and the mixture was concentrated to give $22 \mathrm{f}$ ( $143 \mathrm{mg}, 0.247 \mathrm{mmol}, 67 \%$ ) as a pale brown amorphous solid.

Other derivatives $(\mathbf{2 2 g}, \mathbf{2 2} \mathbf{h})$ were similarly prepared.

Biology. Anti-FXa, Trypsin, FIIa and Plasmin Assay The hydrolysis of chromogenic substrates was assayed by continuously measuring absorbance at $405 \mathrm{~nm}$ at $37^{\circ} \mathrm{C}$ with a microplate reader (SPECTRA max PLUS 384, Molecular Devices, CA, U.S.A.). Reaction mixtures ( $90 \mu 1)$ were prepared in 96-well plates containing enzyme and compounds in reaction buffer ( $50 \mathrm{~mm}$ Tris- $\mathrm{HCl}-150 \mathrm{~mm} \mathrm{NaCl}, \mathrm{pH} 8.4$, as for plasmin: $\mathrm{pH} 7.4)$. Reactions were initiated by the addition of $10 \mu \mathrm{l}$ of substrate and monitored for $5 \mathrm{~min}$. The concentration required to inhibit enzyme activity by $50 \%\left(\mathrm{IC}_{50}\right)$ was estimated from dose-response curves. Enzyme and substrate were used as follows: human FXa (0.5 IU, Enzyme Research Laboratories, Inc., IN, U.S.A.) and S-2222 (4 mM, Daiichi Pure Chemical, Japan); human trypsin ( $750 \mu \mathrm{U}$, Athens Research \& Tech., Inc., GA, U.S.A.) and S-2222 (4 mM, Daiichi Pure Chemical, Japan); human alpha thrombin (1.25NIH u, Enzyme
Research Laboratories, Inc., IN, U.S.A.) and S-2238 (4 mm, Daiichi Pure Chemical, Japan); human plasmin ( $8 \mu \mathrm{g}$, Enzyme Research Laboratories, Inc., IN, U.S.A.) and S-2251 (4 mm, Daiichi Pure Chemical, Japan).

Coagulation Assay Citrated blood samples were collected from healthy male volunteers (Japan SLC). Platelet-poor plasma was prepared by centrifugation at $2000 \times \boldsymbol{g}$ for $10 \mathrm{~min}$ and stored at $-20^{\circ} \mathrm{C}$ until use. Plasma clotting times were determined using a COAGMASTER II (Sankyo, Japan). Prothrombin time (PT) and activated partial thromboplastin time (APTT) were measured using Simplastin Excel (Organon Teknika, NC, U.S.A.) and Platelin LS (Organon Teknika, NC, U.S.A.), respectively. Coagulation times for each compound were compared with coagulation times measured using a distilled water control. Each measurement was performed three times. The concentration required to double the clotting time $\left(\mathrm{CT}_{2}\right)$ was estimated by linear regression analysis using two data points, the two mean values of the concentrations closest to the predicted 2-fold PT.

Acknowledgments We wish to thank Ms. Naoko Suzuki and Ms. Yumiko Fujisawa for their expert technical assistance, and Dr. Ken-ichi Otsuguro for his helpful discussions.

\section{References and Notes}

1) Fujimoto K., Asai F., Tanaka N., JP Patent 2002-088080 (2002).

2) Present address: General Administration Department, Daiichi Sankyo Business Associe Co., Ltd.; 1-8 Nihonbashikomicho, Chuo-ku, Tokyo 103-8541, Japan.

3) Kaiser B., Hauptmann J., Cardiovasc. Drug Rev., 12, 225-236 (1994).

4) Davie E. W., Fujisawa K., Kisiel W., Biochemistry, 30, 10363-10370 (1991).

5) Hara T., Yokoyama A., Tanabe K., Ishihara H., Iwamoto M., Thromb. Haemostasis, 74, 635-639 (1995).

6) Sato K., Kawasaki T., Hisamichi N., Taniuchi Y., Hirayama F., Koshio H., Matsumoto Y., Br. J. Pharmacol., 123, 92-96 (1998).

7) Noguchi T., Tanaka N., Nishimata T., Goto R., Hayakawa M., Sugidachi A., Ogawa T., Asai F., Matsui Y., Fujimoto K., Chem. Pharm. Bull., 54, 163-174 (2006).

8) Noguchi T., Tanaka N., Nishimata T., Goto R., Hayakawa M., Sugidachi A., Ogawa T., Asai F., Ozeki T., Fujimoto K., Chem. Pharm. Bull., 55, 393-402 (2007).

9) Bartoli G., Bosco M., Caretti D., Dalpozzo R., Todesco P. E., J. Org. Chem., 52, 4381- 4384 (1987).

10) Tsunoda T., Yamamiya Y., Ito S., Tetrahedron Lett., 34, 1639-1642 (1993).

11) Oliver J. E., DeMilo A. B., Synthesis, 1975, 321-322 (1975).

12) Bartoli G., Bosco M., Dalpozzo R., Todesco P. E., J. Org. Chem., 51, 3694-3696 (1986).

13) Corey E. J., Bakshi R. K., Shibata S., Chen C. P., Singh V. K., J. Am. Chem. Soc., 109, 7925-7926 (1987).

14) Wallbaum S., Martens J., Tetrahedron Asymmetry, 3, 1475-1504 (1992).

15) Brandstetter H., Kuhne A., Bode W., Huber R., von der Saal W., Wirthensohn K., Engh R. A., J. Biol. Chem., 271, 29988-29992 (1996).

16) Adler M., Davey D. D., Phillips G. B., Kim S.-H., Jancarik J., Rumennik G., Light D. R., Whitlow M., Biochemistry, 39, 12534-12542 (2000).

17) Maignan S., Guilloteau J.-P., Pouzieux S., Choi-Sledeski Y. M., Becker M. R., Klein S. I., Ewing W. R., Pauls H. W., Spada A. P., Mikol V., J. Med. Chem., 43, 3226-3232 (2000).

18) Phillips G., Guilford W. J., Buckman B. O., Davey D. D., Eagen K. A., Koovakkat S., Liang A., McCarrick M., Mohan R., Ng H. P., Pinkerton M., Subramanyam B., Ho E., Trinh L., Whitlow M., Wu S., Xu W., Morrissey M. M., J. Med. Chem., 45, 2484-2493 (2002).

19) Willardsen J. A., Dudley D. A., Cody W. L., Chi L., McClanahan T. B., Mertz T. E., Potoczak R. E., Narasimhan L. S., Holland D. R., Rapundalo S. T., Edmunds J. J., J. Med. Chem., 47, 4089—4099 (2004). 